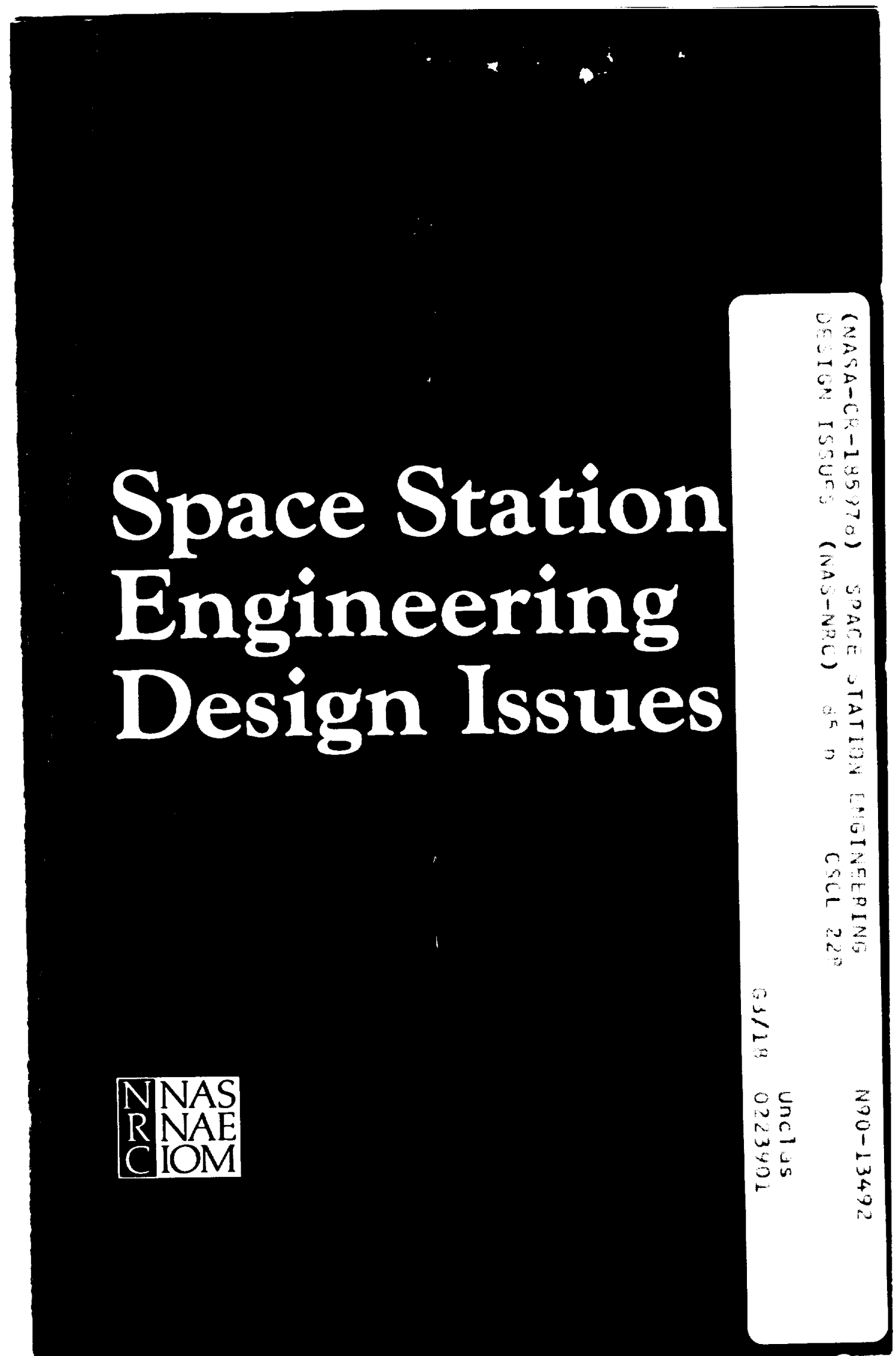


-

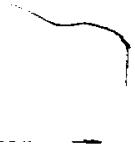




\title{
SPACE STATION ENGINEERING DESIGN ISSUES
}

\author{
Report of a Workshop
}

November 7-11, 1988

Irvine, California

Workshop Committee on Space Station

Engineering Design Issues

Aeronautics and Space Engineering Board

Commission on Engineering and Technical Systems

National Research Council

NATIONAL ACADEMY PRESS

Washington, D.C. 1989 
NOTICE: The project that is the subject of this report was approved by the Governing Board of the National Research Council, whose members are drawn from the councils of the National Academy of Sciences, the National Academy of Engineering, and the Institute of Medicine. The members of the committee responsible for the report were chosen for their special competences and with regard for appropriate balance.

This report has been reviewed by a group other than the authors according to procedures approved by a Report Review Committee consisting of members of the National Academy of Sciences, the National Academy of Engineering, and the Institute of Medicine.

The National Academy of Sciences is a private, nonprofit, self-perpetuating society of distinguished scholars engaged in scientific and engineering research, dedicated to the furtherance of science and technology and their use for the general welfare. Upon the authority of the charter granted to it by the Congress in 1863 , the Academy has a mandate that requires it to advise the federal government on scientific and technical matters. Dr. Frank Press is president of the National Academy of Sciences.

The National Academy of Engineering was established in 1964, under the charter of the National Academy of Sciences, as a parallel organization of outstanding engineers. It is autonomous in its administration and in the selection of its members, sharing with the National Academy of Sciences the responsibility for advising the federal government. The National Academy of Engineering also sponson engineering programs aimed at meeting national needs, encourages education and research, and recognizes the superior achievements of engineers. Dr. Robert $M$ White is president of the National Academy of Engineering.

The Institute of Medicine was established in 1970 by the National Academy of Sciences to secure the services of eminent members of appropriate professions in the examination of policy matters pertaining to the health of the public. The Institute acts under the responsibility given to the National Academy of Sciences by its congressional charter to be an adviser to the federal government and, upon its own initiative, to identify issues of medical care, research, and education. Dr. Samuel 0 . Thier is president of the Institute of Medicine.

The National Research Council was organized by the National Academy of Science in 1916 to associate the broad community of science and technology with the Academy's purposes of furthering knowledge and advising the federal government. Functioning in accordance with general policies determined by the Academy, the Council has become the principal operating agency of both the National Academy of Sciences and the National Academy of Engineering in providing services to the government, the public, and the scientific and engineering communities. The Council is administered jointly by both Academies and the Institute of Medicine. Dr. Frank Press and Dr. Robert $M$. White are chairman and vice-chairman, respectively, of the National Research Council.

This report and the study on which it is based were supported by Contract NASW-4003 between the National Aeronautics and Space Administration and the National Academy of Sciences.

Additional copies of this publication are available from:

National Academy Press

2101 Constitution Avenue, N.W.

Washington, DC 20418

Library of Congress Catalog Card No. 89-62778

Printed in the United States of America 


\section{Workshop Committee on Space Station Engineering Design Issues

DUANE T. McRUER (Chairman), President, Systems Technology, Inc.

BARRY W. BOEHM, Chief Scientist, TRW Defense Systems Group

DANIEL B. DEBRA, Professor of Aeronautics and Astronautics, Stanford University

C. CORDELL GREEN, Director, Kestrel Institute

RICHARD C. HENRY, Lieutenant General, U.S. Air Force (Retired)

PAUL D. MAYCOCK, President, PV Energy Systems, Inc. $P Q 0375 / 2$

JOHN H. MCELROY, Dean of Engineering, University of Texas--Arlington

CHESTER M. PIERCE, Professor of Psychiatry, Harvard University

THOMAS P. STAFFORD, Lieutenant General, U.S. Air Force (Retired)

LAURENCE R. YOUNG, Professor of Aeronautics and ? Astronautics, and Director of the Man-Vehicle Laboratory, Massachusetts Institute of Technology

\section{ASEB Technical Liaison}

BYRON K. LiCHTENBERG, President, Payload Systems, Inc.

Staff

RICHARD M. OBERMANN, Study Director

JENNIFER T. ESTEP, Administrative Secretary 


\section{Aeronautics and Space Engineering Board}

ALBERTUS D. WELLIVER (Chairman), Vice President, The Boeing Company

EUGENE E. COVERT (Vice-Chairman), Professor and Head, Department of Aeronautics and Astronautics, Massachusetts Institute of Technology

ELIZABETH E. BAILEY, Dean, Graduate School of Industrial Administration, Carnegie-Mellon University

JAMES M. BEGGS, Consultant

RICHARD G. BRADLEY, Director, Aerospace Technology, General Dynamics Corporation

BERNARD BUDIANSKY, Gordon McKay Professor of Structural Mechanics, Abbott and James Lawrence Professor of Engineering, Harvard University

ROBERT H. CANNON, JR., Charles Lee Powell Professor and Chairman, Department of Aeronautics and Astronautics, Stanford University

RICHARD W. HESSELBACHER, Executive Vice President-Programs, Fairchild Space Company

BYRON K. LICHTENBERG, President, Payload Systems, Inc.

ROBERT G. LOEWY, Institute Professor, Mechanical and Aerospace Sciences, Rensselaer Polytechnic Institute

STANLEY MARTIN, JR., Technical Director, Bell-Boeing Joint Program Office

JOHN H. McELROY, Dean of Engineering, University of Texas-Arlington

DUANE T. McRUER, President, Systems Technology, Inc.

GARNER W. MILLER, Senior Vice President, Maintenance and Engineering, USAir

GEORGE W. MORGENTHALER, Associate Dean for Research, College of Engineering and Applied Science, Professor and Chairman, Department of Aerospace Engineering Sciences, University of Colorado

HARVEY O. NAY, Director of Engineering, Piper Aircraft Corporation

FRANK E. PICKERING, Vice President and General Manager, Aircraft Engines Engineering Division, General Electric Company

R. BYRON PIPES, Dean of Engineering, University of Delaware 
ANATOL ROSHKO, Theodore Von Karman Professor of Aeronautics, California Institute of Technology

RICHARD S. SHEVELL, Professor, Department of Aeronautics and Astronautics, Stanford University

THOMAS P. STAFFORD, Lieutenant General, U.S. Air Force (Retired)

Liaison Member

FRANKLIN K. MOORE, Joseph C. Ford Professor of Mechanical Engineering, Cornell University (Sabbatical at NASA

Headquarters through September 1989)

\section{ASEB Staff}

ROBERT H. KORKEGI, Director

JOANN C. CLAYTON, Senior Staff Officer

RICHARD M. OBERMANN, Senior Staff Officer

ANNA L. FARRAR, Administrative Assistant

JENNIFER T. ESTEP, Administrative Secretary

ELIZABETH LEE, Senior Secretary 



\section{Contents}

Executive Summary, 1

1. Introduction, 9

- Background, 9

- Approach, 11

2. General Design Issues, 15

- Crew Safety, 15

- Ground Verification of Elements, Assemblies, and Operations, 16

- Reliance on Models and Modeling for Verification, 16

- On-Orbit Assembly, Integration, and Verification, 16

- Sof tware Environment, 17

- Resource Adequacy and Allocation for Space Station Assembly and Operations, 17

- "Insurance" Alternatives for Flight-Critical Systems, 18

- Standards and Commonality, 18

- Design for Future Refurbishment, 18

3. Issues Related to Utilization and Operations Requirements for the Space Station, 19

- Design Reference Missions, 21

- Assembly Phase Requirements, 22

- Assembly Sequence, 22

- Premature Flight Telerobotic Servicer Launch, 22

- Assembly Phase Utilization, 23

- Mature Utilization and Operations Requirements, 24

- Microgravity Environment, 24

- Crew Utilization, 25

- Commonality, 27

- Logistical Resupply, 27

- Contingency Planning, 28

- Evolutionary Phase Requirements, 29

- Essential Evolutionary Capabilities, 29

- Heroic Evolution, 29

PRECEDING FAGE BLANK NOT FILMED

vii 7 Vi MTEMTOHALLY RLANK 
4. Issues Related to Systems Requirements and Design, 31

- Systems Architectural Concerns, 31

- Systems Requirements, 31

- Systems Interactions, 32

- Allocation of Systems Requirement Impacts, 33

- Modeling, 34

- Growth Flexibility, 35

- Specific Systems Issues, 35

- Software and Data Management, 35

- Overview, 35

- Software Risk Management, 37

- Software Development Schedules and Uncertainties in Application Sof tware Requirements, 38

- Software Support Environment, 39

- Software Design for Supportability, 39

- Sof tware Integration and Verification, 40

- Space Station Information System Services, 41

- Communications and Tracking, 42

- End-to-End Considerations, 42

- Electromagnetic Interference, 43

- Automation and Robotics, 45

- Premature Flight Telerobotic Servicer (FTS) Launch, 45

- Solution-Driven Versus Problem-Driven Approaches to Automation Initiatives, 45

- Advanced Automation Targets and Products, 46

- Advanced Automation and Robotics Plan, 46

- Electrical Power System, 47

- Thermal Control System, 48

- Environmental Control and Life Support System, Man Systems, and Extravehicular Activity System, 49

- Overview, 49

- Microbial and Toxin Control in the Space Station, 49

- In-Space Testing of Life Support Systems, 50

- Medical Evacuation, 50

- Human Factors and Habitability, 50

- Radiation, 51

- Extravehicular Activity, 51

- Fluid Management System, 51 
5. Management Issues Relevant to Design, 53

- Impact of the Internal NASA Culture on the Space Station Program, 54

- Background, 54

o Impact of Management Complexities on the Space Station Technical Program, 55

- Impact of Program Instabilities on the Space Station Technical Program, 56

- Management of Free-Flying Platforms, 57

- Associate Contractor Relationships, 58

- Technical Management Process, 58

- System Specification, 59

- Integration and Verification Management Plan, 59

- Communications and Data Systems, 60

Supplementary Discussion, 61

- Materials, 61

- Health Maintenance, 62

- Toxic Materials Handling, 62

- Potential Incompatibility of User Requirements, 63

- Crew Safety, 63

Appendixes

A. Letter from the NASA Office of Space Station, 65

B. Statement of Task, 67

C. Presenters at the Workshop, 69

Abbreviations/Symbols, 71

Acronyms, 73

Selected Material Used at the Workshop, 75 
- 


\section{Executive Summary}

The Space Station Freedom program is the next major U.S. manned space initiative. It has as its objective the establishment of a permanently manned facility in low earth orbit. The facility is intended to be used for a range of activities and to accommodate a number of alternative evolutionary growth paths. The Space Station also is envisioned, by the National Aeronautics and Space Administration (NASA) and others, as an essential element in the recovery and maintenance of U.S. leadership in civil space activities and as an opportunity for increased international cooperation.

In early 1987, the NASA Associate Administrator for Space Station indicated an interest in having the National Research Council (NRC) examine the Space Station program with a view toward identifying critical engineering issues related to the design and operation of the station. This activity was preempted by a 1987 NRC study of the Space Station program undertaken at the request of the President's Assistant for National Security Affairs, the Director of the Office of Management and Budget, the President's Science Advisor, and the Administrator of NASA. However, in early 1988, the Associate Administrator for Space Station submitted a formal request for an NRC workshop to identify and prioritize Space Station engineering issues. A week-long, intensive workshop was held on November 7-11, 1988, at the Arnold and Mabel Beckman Center of the National Academy of Sciences and the National Academy of Engineering in Irvine, California; this report summarizes the main findings and recommendations of that workshop, and reflects the views and opinions of the workshop committee.

The workshop was not a technical audit of the program. The report thus does not focus on identifying all the good features 
of the Space Station program, although the committee has noted some of these aspects at various points in the report. In addition, a number of areas of possible concern, such as the effect of the space environment on Space Station materials and structures, were not represented in the briefings to the committee or by its expertise. Consequently, the issues identified in this report should not be construed as an exhaustive set of concerns, but rather those that appeared most important to the committee based on its necessarily limited review of the Space Station in November 1988. Finally, the limited time available to the committee made it impossible to do a more detailed prioritization of the issues identified in this report, especially across subsystems.

Major issues identified by the workshop committee were those related to general design, utilization and operations requirements, and specific Space Station systems and subsystems. The committee also raised a number of management issues that it believes could have an important impact on Space Station design and operation.

\section{GENERAL DESIGN ISSUES}

1. The major issue of assuring the safe return of the Space Station's crew under emergency conditions has not been addressed in the Space Station concept presented to the committee; the committee believes a crew emergency rescue vehicle is required.

2. The allocation of verification activities and/or checkout of subsystems and assemblies (including sof tware) between those activities that are ground-based and those that will be done on orbit is an important unresolved issue.

3. There is an extensive and perhaps excessive reliance on modeling of systems and software for verification in lieu of ground or flight testing.

4. The current schedule for the assembly of the Space Station on orbit appears incompatible with integration and verification requirements.

5. The committee believes NASA is to be commended for recognizing the criticality of sof tware and data management to the Space Station program and for taking a proactive approach to addressing software issues through investments in a program-wide Sof tware Support Environment (SSE) and Technical and Management Information System (TMIS). However, it is 
concerned that the SSE as mandated may be relatively inflexible and restrictive for some program purposes, such as real-time dynamic systems modeling.

6. The committee is concerned that there are inadequate margins and in some cases actual resource shortfalls (e.g., power, existing post-Challenger Shuttle payload weight margins for assembly flights) at this early stage of the Space Station program.

7. Preliminary design considerations for system alternatives to serve as "insurance" for flight-critical systems (e.g., electrical power, thermal control, life support) are not yet being developed.

8. Questions of common measurement standards, commonality of tools and equipment, and so forth do not appear to have been resolved in a concrete fashion that is consistent with the planned long life of the Station.

9. Hardware and software provisions for future expansion or evolution of the Space Station are under study by the Space Station program. However, provisions for refurbishment, repair, and rebuilding over a multiyear time span do not appear to have received the same attention.

\section{ISSUES RELATED TO UTILIZATION AND OPERATIONS REQUIREMENTS}

1. The committee believes that the general issue of the compatibility of all the planned/potential users of the Space Station is very important and needs to receive more attention.

2. The current Design Reference Missions, which show the Station resources to be more or less in balance, may not allow an accurate assessment of user and operator needs. Development of Design Reference Missions should be carried out with the intention of probing the design's weaknesses rather than demonstrating its strengths.

3. Further, it was evident to the committee that Shuttle and Spacelab precedents were used in developing procedures for user experimentation on the Space Station. More innovative processes are needed to improve responsiveness to user needs consistent with safe and effective Station operations.

4. The committee is concerned that the Space Station's design, assembly, and operation may have become unduly constrained by such things as existing post-Challenger Shuttle 
performance limitations, certain congressional directives, and some user requirements. It is important that the justification for each constraint be firmly established and its impact on the program clearly understood.

5. A premature demonstration flight of the Flight Telerobotic Servicer (FTS) concept and early manifesting of the FTS in the Space Station assembly sequence is likely to have a contrary effect to the intended objective of advancing automation and robotics.

6. The current program plan, which does not accommodate the animal/plant centrifuge in the U.S. laboratory module, and which proposes to assemble and check out the life sciences facilities on orbit, does not appear to meet the requirements established by NASA and NRC advisory committees.

7. A particularly sensitive issue to the Space Station life science and materials science users is the production, transmission, and absorption of vibrations at frequencies that could damage the microgravity processes under study. Until the Space Station program tackles the vibration transmission issue squarely, such disturbances will have to be treated empirically, with a resulting awkwardness in layout and operations.

8. The protection of the amount of crew time available for user activities is vulnerable to erosion as maintenance and operations requirements are better defined.

9. The committee is concerned that the Space Station program has no provision for unmanned resupply of the U.S. modules (e.g., facilities for unmanned rendezvous and docking).

\section{SELECTED ISSUES RELATED TO SYSTEM REQUIREMENTS AND DESIGN}

1. The committee did not find as ready an awareness of the impact of system interactions as it believes is desirable at this stage in the Space Station development program.

\section{Software and Data Management}

2. No software risk management plan exists, although major software risk factors exist.

3. The current sof tware schedule emphasizes early completion of software requirements documents, which then become baselined and more difficult to modify. The early 
schedule should preferably emphasize execution of risk management plans and resolution of risk items before sof tware requirements are "cast in concrete."

4. The coordination of the Space Station Information System (SSIS) with the other data distribution systems proposed by NASA user organizations does not appear to be well defined.

\section{Communications and Tracking}

5. No end-to-end perspective in communications and tracking was evident or presented to the committee.

6. The committee is concerned that all communications to and from the Space Station will flow via the Tracking and Data Relay Satellite System.

7. The Space Station is dependent on many communication links for its successful operation; the vulnerability of these links to deliberate or inadvertent interference or access is a continuing concern.

8. The relative inaccessibility of the Space Station and the relatively meager resources that will be available to the crew heighten the need for special attention to the potential problem of electromagnetic interference at the Station.

\section{Automation and Robotics}

9. More emphasis is needed on advanced automation tools and capabilities to improve the efficiency of Space Station user operations.

10. There is no clear vision of the spectrum of applications to be supported by the FTS, particularly given the existence of the Canadian Mobile Servicing System.

\section{Electrical Power System}

11. Power adequacy for the Phase 1 Space Station is of concern to the committee.

12. There is need for a management review of all utilities: electricity, heat, water, and so forth.

\section{Thermal Control System}

13. More attention should be given to the analysis of thermal control system trade-offs. 
Environmental Control and Life Support System, Man Systems. Extravehicular Activity System

14. Microbial and toxin control in the Space Station is a continuing area of concern.

15. The committee is concerned that sufficient in-space testing be done on the planned Space Station life support system.

16. The requirements for medical evacuation and its associated problems need to be addressed.

17. The committee believes that the issue of Space Station crew efficiency over time is an important one, with implications for operational timelines and crew schedules.

\section{Fluid Management System}

18. The control and handling of waste liquids and gases, as well as hazardous materials used in the laboratories, need to be addressed vigorously.

\section{MANAGEMENT ISSUES RELEVANT TO DESIGN}

1. The committee believes that the Space Station management structure has complicated the Space Station development task.

2. Budgetary uncertainties, certain congressional directives, and operational limitations of the existing post-Challenger Shuttle have produced program instabilities that may have increased design complexity and reduced design conservatism.

3. The retention of the polar platform within the Space Station program has further complicated the program's management task.

4. The committee is concerned that a disciplined and contractually binding process for integration is not evident; one needs to be established early in the program.

5. A Space Station System Specification is needed to contractually define the system and provide a basis to effect change discipline and change control.

6. While there is considerable work under way, there does not appear to be a strong and crisp integration and verification management plan and architecture in place. 
7. The communications and data systems for the Space Station pass through elements that are developed by up to four separate NASA offices, with little evidence that adequate oversight is being provided by NASA. Thus, the potential exists for errors to be made (notably those of omission) even among well-intentioned, skilled organizations. 



\section{Introduction}

The Space Station Freedom program has as its objective the establishment of a permanently manned facility in low earth orbit. The facility is intended to be used for a range of activities and to accommodate a number of alternative evolutionary growth paths. The Space Station also is envisioned, by the National Aeronautics and Space Administration (NASA) and others, as an essential element in the recovery and maintenance of U.S. leadership in civil space activities and as an opportunity for increased international cooperation.

Table 1 lists the major intended attributes of the Space Station Freedom manned base (the program also includes polar orbiting platforms and a co-orbiting man-tended free-flyer). The Phase 1 Space Station is planned to be a facility with the potential to support activities in microgravity materials research, fluid physics, crystal growth, biomedical studies, human habituation studies, and astrophysical and earth observations. The station would also support in-space technology experiments and development efforts.

\section{BACKGROUND}

There have been a number of external reviews of the Space Station Freedom program. The National Research Council (NRC), at the request of the President's Assistant for National Security Affairs, the Director of the Office of Management and Budget, the President's Science Advisor, and the Administrator of the National Aeronautics and Space Administration, undertook a study of the Space Station program in 1987. Prior to that

PRECEDING PAGE BLANK NOT FILMER6E, 8 INTENIIONALIY RIAMK 
Table 1 Intended Attributes of Space Station Freedom Manned Base

\section{A Permanently Occupied Spacecraft}

A Research Laboratory

An Observational Platform

Very High User Power ( $45 \mathrm{~kW})$

Very Low Quasi-Steady Microgravity Accelerations

An Optimized Spacecraft Attitude

High Data Rate (300 Mbps)

Weekly Extravehicular Activity (6 hours/week)

An Internal Environment of One Standard Atmosphere

Clean External Environment (Gases, Condensibles, EMI)

\section{A Payload Pointing System}

Large Annual Upmass Capability $(50,000 \mathrm{~kg} / \mathrm{yr})$

Accommodations for Space Station Evolution
The spacecraft would have a crew of eight and a facility lifetime of $\mathbf{3 0}$ years; it is designed to conduct the operational and medical research required to support long-term manned space exploration objectives.

More than 120 standard equipment racks would be available to hold scientific research equipment.

The platform could hold four to five large experiment footprints of $10 \mathrm{~m}^{2}$ for long (up to 30 years) observation times.

Such power levels are required to operate experimenta; they are especially neceseary to power furnaces, and so forth, for materials research.

Accelerations of $1 \times 10^{-6} \mathrm{~g}$ (at $\mathrm{f}<0.1 \mathrm{~Hz}$ ) are critical for materiala research in order to force diffusion-dominated processes.

Designed to benenit both laboratory and observational experiments (LVLH $=+/-55^{\circ}+/-2.5^{\circ}$ stability; $<.02 \%$ (sec stabilization rate), this attitude keeps the residual microgravity acceleration vector in a near-constant direction for materials research. It also provides sufficient pointing orientation for many observing instruments. It is accomplished without disturbing thruster firings, by using control moment gyros.

This rate will support modern research needs for high resolution and high-speed image transfer.

EVAs would be available to users for external, observational experiment resupply (cryogens, film, etc.) as well as for servicing and repair.

This internal environment is critical, since it allows researchers to draw on decades of ground-based life science data.

A clean environment is necessary for the conduct of optical and plasma observations.

It will handle large payloads ( $3 \mathrm{~m}$ in diameter by $4 \mathrm{~m}$ long, $6,000 \mathrm{~kg}$ mass), providing $30 \mathrm{arc} \mathrm{sec}$ of inertial pointing for precision obervations.

A large upmass capability is needed to provide resupply and experiment change-out for research.

The Station is designed to allow more power, crew, laboratory space, and attached payload space to be added. 
activity, the Aeronautics and Space Engineering Board of the NRC conducted examinations of various aspects of the Space Station program during 1984 and 1985 , including its maintainability, program performance, onboard command and control, solar thermodynamics research and technology, and research and technology in space.

In early 1987, the NASA Associate Administrator for Space Station indicated an interest in having the NRC examine the Space Station program with a view toward identifying critical engineering issues related to the station's design and operation. This activity was preempted by the aforementioned study for the White House, et al. However, in early 1988 the NASA Office Space Station submitted a formal request for an NRC workshop to identify and prioritize Space Station engineering issues. (See Appendix A) The workshop was held on November $7-11,1988$, at the Beckman Center in Irvine, California; this report summarizes the main findings of that event, and reflects the views and opinions of the workshop committee.

\section{APPROACH}

Following formal acceptance of NASA's request by the NRC's Governing Board, an ad hoc committee was formed by the Aeronautics and Space Engineering Board to conduct the workshop. The members of the committee were chosen for their expertise in the areas of space systems, space structures, guidance and control, operations and management, space transportation, power, communications, space medicine and life sciences, robotics, sof tware development and management, utilization, and space applications. Prior to the workshop, the committee was supplied with extensive background materials. The workshop itself was an intensive, four-and-a-half day review of the engineering and operational aspects of the planned Space Station, with some sessions lasting until nearly midnight. Nonetheless, the magnitude of the program under review coupled with the limited time available to conduct the review, make several caveats necessary:

- The workshop was not a technical audit. Most of the committee's information was based on views of the Space Station program and design plans as of November 1988, derived primarily 
from briefings presented at the workshop by Level I and Level II personnel of the NASA Space Station program.

- Some areas of possible concern, such as materials and the effect of the space environment thereon, were not represented in the briefings or by the expertise of the committee. Some thoughts of the committee concerning these areas are included in the Supplementary Discussion section of this report.

- The workshop report has not attempted to identify all the good features of the Space Station program. The committee has noted some of these aspects at various points in the report, but most of its energies have been directed--in accordance with its task statement (see Appendix B)--toward the identification of issues.

- The limited time available to the committee made it impossible to do a more detailed prioritization of the issues identified in this report, especially across subsystems. However, the committee does believe that all of the issues identified in this report will require resolution for the Space Station program to be successful.

Consequently, the issues identified in this report should not be construed as an exhaustive set of concerns, but rather those that appeared most important to the committee based on its necessarily limited review of the Space Station program. Furthermore, the committee was not asked to address the desirability of developing a Space Station; President Reagan's 1984 decision was taken as the committee's point of departure.

In its deliberations the committee has searched for the inconsistent, the overly optimistic, and for those areas that, if they continue in the direction they are currently headed, are likely to be troublesome later. The committee used two basic principles in its evaluation: (1) early recognition and correction of potential problems is the most efficient and least costly approach, and (2) flexibility in design and "insurance" provided by alternative approaches can yield major downstream dividends, especially when coping with unexpected or unanticipated events.

The following sections of the report present the workshop committee's major findings and recommendations. A number of general design issues are discussed first. Next, issues related to Space Station utilization and operations requirements are identified, followed by issues concerning specific Space Station systems and subsystems. Finally, a 
number of management issues that the committee believes could have an important impact on Space Station design and operation are discussed. 



\section{General Design Issues}

A number of issues emerged from the committee's review that it believes can have an exceptional impact on the design and ultimate success of the Space Station. These issues either cross subsystem boundaries or have facets or attributes that are common to several subsystems. Because of their overarching nature, they are presented first.

\section{CREW SAFETY}

The major issue of assuring the safe return of the Space Station's crew under emergency conditions has not been addressed in the Space Station concept presented at the November 1988 workshop. The committee awaits with interest the results of the Crew Emergency Rescue Vehicle (CERV) studies currently under way, but it is reluctant to rely on the assurances that onboard redundancy will be sufficient to handle any and all possible life-threatening malfunctions. The earlier NRC Committee on Space Station believed that "NASA should establish a mandatory requirement for a crew emergency rescue vehicle and should consider its use, on a man-rated expendable launch vehicle, as a backup means of manned access to the Space Station" (Report of the Committee on the Space Station of the National Research Council, p. 7, 1987). The workshop committee agrees with that recommendation.

\section{PRECEDING PAGE BLANK NOT FILMED}




\section{GROUND VERIFICATION OF ELEMENTS, ASSEMBLIES, AND OPERATIONS}

The allocation of verification and/or checkout of subsystems and assemblies (including sof tware) between those activities that are ground based and those that will be done on orbit is another important unresolved issue. Furthermore, the degree to which flight equipment will be duplicated for checkout, verification, spares purposes, and so forth, is only vaguely defined in the current Space Station program plans, as briefed to the committee. A verification strategy, involving ground checkout of flight hardware, simulation, dedicated ground test hardware, and on-orbit verification is sorely needed and must be specified.

\section{RELIANCE ON MODELS AND MODELING FOR VERIFICATION}

There is an extensive and perhaps excessive reliance on modeling of systems and sof tware for verification in lieu of ground or flight testing. In addition, backup plans for handling the on-orbit problem-resolution activities that may be needed in the absence of prior ground-based verification are not in place and do not appear to have been considered in sufficient depth.

\section{ON-ORBIT ASSEMBLY, INTEGRATION, AND VERIFICATION}

The current schedule for the on-orbit assembly of the Space Station appears incompatible with integration and verification (I\&V) requirements.

The assembly period between achievement of a man-tended capability and a permanently manned capability (PMC) is scheduled to last about one year. However, on-orbit I\&V implies considerable testing between assembly stages to establish operating envelopes.

Each stage of Space Station assembly results in a complete spacecraft on orbit. The plans for on-orbit I\&V likely will require special coordination to insure that the designers (e.g., the structural dynamicists and control engineers) who are most affected have the data needed to verify their models 
in time to improve their analysis of subsequent Space Station increments.

Establishment of an assembly plateau after achieving a man-tended capability for thorough on-orbit I\&V could enable incorporation of lessons learned and resulting product modifications into flight packages that would be deployed between the man-tended and permanently manned capability. Another plateau after the permanently manned capability could enable improved efficiency to be achieved in the full Phase 1 configuration.

A major on-orbit assembly problem is the coordination of assembly operations between the Shuttle and the protostation elements. Some of the crucial issues involve joint crew activities by Shuttle and Space Station astronauts, the nature of the coupled dynamic behavior during assembly, appropriate docking/berthing strategies, and assembly sequence resiliency in an environment of Shuttle mass margin uncertainty.

\section{SOFTWARE ENVIRONMENT}

While the committee believes NASA is to be commended for recognizing the criticality of software and data management to the Space Station program and for taking a proactive approach to addressing software issues through investment in a program-wide Sof tware Support Environment and Technical and Management Information System, it is concerned that the Sof tware Support Environment as mandated may be relatively inflexible and restrictive for some program purposes, such as real-time dynamic systems modeling. Strict adherence to the Software Support Environment is likely to create an enormous make-work chore for many activities that should not require such adherence.

\section{RESOURCE ADEQUACY AND ALLOCATION FOR SPACE STATION ASSEMBLY AND OPERATIONS}

Critical resources for assembly of the Space Station include existing post-Challenger Shuttle payload weight and volume margins on individual assembly flights. Extravehicular activity time margins and Shuttle stay-times for assembly flights are additional resources that must be traded off against Shuttle payload weights. Electrical power, pressurized 
laboratory volume, external payload attachment points and "real estate" on the truss structure, and data transmission capacities are among the resources critical to the successful operation of the Space Station.

The committee is concerned that there are inadequate margins and in some cases actual resource shortfalls at this early stage of the Space Station program.

\section{"INSURANCE" ALTERNATIVES FOR FLIGHT-CRITICAL SYSTEMS}

Preliminary design considerations for system alternatives to serve as "insurance" for flight-critical systems (e.g., electrical power, thermal control, life support) are not yet being developed. The existence of such alternative design possibilities could reduce program risk or help cover projected shortfalls. While the Space Station design might not be at the level of maturity to do this yet, a strategy for developing "insurance" system possibilities is needed to assure that this activity is not neglected.

\section{STANDARDS AND COMMONALITY}

Questions of common measurement standards, commonality of tools and equipment, and so forth do not appear to have been resolved in a concrete fashion that is consistent with the planned long life of the Station. This issue will be discussed further in chapter 3 .

\section{DESIGN FOR FUTURE REFURBISHMENT}

Hardware and software provisions for future expansion or evolution of the Space Station are under study by the Space Station program. However, provisions for refurbishment, repair, and rebuilding over a multiyear time span do not appear to have received the same attention. Such considerations are important for the long-term viability of the Space Station. 


\section{Issues Related to Utilization and Operations Requirements for the Space Station}

The overall Space Station utilization and operations requirements stem from the need to satisfy a multiplicity of currently planned as well as potential evolutionary purposes. The Phase 1 Space Station is planned to be a facility supporting such activities as microgravity materials research, biomedical studies, and human habituation studies. It also will accommodate externally attached scientific payloads to a limited extent.

The evolution of the Space Station beyond Phase 1 could move in any of a number of directions, including development into a transportation node for manned lunar and/or planetary missions, an assembly facility for the construction of large spacecraft or other in-space inf rastructure, a depot for servicing space assets, or perhaps even a dedicated life sciences or materials science laboratory. The Space Station design is intended to allow flexibility in utilization and operation as it evolves.

At present, the Space Station is being designed principally to serve users from the major space science and technology disciplines. On the manned base facility, the key resources are the external attachment points with associated power and data link capabilities, and the internal pressurized

volume--first in the two pressurized U.S. modules and later in the Japanese and European Space Agency modules. The major users of the pressurized module volume will be the materials science and life science communities. Earth observation requirements are to be met mainly by the Polar Orbiting Platform.

Space Station Freedom is intended to be an international facility--in planning, management, and operation. The international space science community, as represented by the 
International Forum on the Scientific Uses of the Space Station has strongly supported a shared international facility with commonality of interfaces and sharing of major facilities, independent of agency boundaries. To the extent that the Space Station Memoranda of Understanding and the management structure support this concept, meaningful international cooperation can take place. The development of an integrated design with well-defined interfaces across the U.S.- and non-U.S.-provided facilities will require continued attention.

The overall Station layout and the detailed planning for utilization of the transverse boom and the lab modules are generally consistent with the emphasis on two new contributions of the Station: long durations and sufficient pressurized volume for significant microgravity research. Layout and planning are also consistent with the intended attributes for the Station summarized in Table 1. However, several attributes listed in Table 1 may themselves be inconsistent or even partially competitive. For example, some crew operations may have to be restricted to avoid disturbing the low levels of microgravity $\left(10^{-6} \mathrm{~g}\right)$ required for classes of proposed experiments. Thus, while some previous studies have indicated that the same laboratory can be used for both materials and biological research, adequate attention must be paid to the impacts of such cooperative or simultaneous operations--for example, the need for vibration and contaminant isolation.

The committee believes that the general issue of the compatibility of all the planned/potential users of the Space Station is very important and needs to receive more attention. Moreover, even though requirements in a particular discipline may not be completely defined, the ultimate possible extent of the requirements may be crucial inputs to architectural decisions being made now (e.g., compatibility of animal experimentation with planned Space Station environmental and decontamination provisions).

With respect to the evolution of the Space Station, its potential utilization as a base for future manned missions does not present requirements at this time that are specific enough to influence the detailed design of the Phase 1 Space Station. Only when and if requirements for the assembly and launch of planetary or lunar base missions are defined can the appropriate Space Station evolutionary path to support them also be defined. However, continued care must be taken during the Phase 1 Space Station design process so as not to preclude 
such use by arrangement of the Phase 1 geometry or other characteristics.

The following sections identify specific issues raised by the workshop committee regarding Space Station utilization and operations requirements. Issues include those associated with Design Reference Missions, which illuminate the implications of user and operator requirements for Station design, assembly phase requirements, mature utilization and operations requirements, and evolutionary phase requirements.

\section{DESIGN REFERENCE MISSIONS}

In order to guide the requirements generation process and to check the adequacy of the current Space Station design, some reasonable operational scenarios along with representative experiments to be conducted are required. For the second purpose a set of "Design Reference Missions" is being developed and used to identify the strengths and weaknesses of the Space Station design. These Design Reference Missions are intended to be both representative and comprehensive. However, it is by no means clear that the missions chosen are really design drivers that illuminate the most difficult features and/or incompatibilities in the design. Clearly, the nature of the Design Reference Missions used is an important determinant of the success of the paper evaluation of the design. The weak points will only be understood by returning to each scientific discipline's list of approved missions and deliberately selecting mission combinations that stress the Station's resources, especially data capacities, power, and crew time.

The committee is concerned that the current exercises, which show the Station's resources to be more or less in balance, with the first limitation coming from power, may not allow an accurate assessment of such user and operator needs as: removal of bottlenecks in multiple video links, provision of separate work stations, and additional crew accessibility. Development of Design Reference Missions should be carried out with the intention of probing the design's weaknesses rather than demonstrating its strengths. For example, the committee questions the claim that the volume available--either inside the pressurized modules and nodes or on the external boom--is sufficient to accommodate the users who could benefit from the currently planned power level, data capacities, or crew time. 
Further, it was evident to the committee that Shuttle and Spacelab precedents were used in developing procedures for user experimentation on the Space Station. Precedents that are troublesome include the Space Shuttle and Spacelab procedures related to integration and verification of hardware and software for user experiments, which require very long lead times. More innovative processes are needed to improve responsiveness to user needs consistent with safe and effective Station operations.

\section{ASSEMBLY PHASE REQUIREMENTS}

\section{Assembly Sequence}

The assembly sequence for the Phase 1 Space Station is constrained in several important ways. Among these are existing post-Challenger Shuttle performance limitations that have an impact on Space Station assembly by limiting the allowable payload mass and/or volume and maximum extravehicular activity time by the crew per Shuttle flight. Another set of constraints are those imposed by selected congressional directives. The directives deal with launch of the Flight Telerobotic Servicer (FTS) (a robotic arm assembly) by the second assembly flight, early utilization of the U.S. laboratory module for man-tended materials processing prior to the Space Station's permanently manned phase, and the location of life science facilities on the Station. All of these constraints have increased the design and operational complexity of the Space Station. The committee is concerned that the Space Station's design, assembly, and operation may have become unduly constrained and believes it is important that the justification for each constraint be firmly established and its impact on the program clearly understood.

\section{Premature Flight Telerobotic Servicer Launch}

The objective of advancing automation and robotics to improve scientific utilization of the Space Station as well as to increase the efficiency of operations is laudable and should, in the long run, provide significant productivity benefits. However, a premature demonstration flight of the FTS concept and early manifesting of the FTS in the Space Station assembly sequence unfortunately is likely to have just the 
contrary effect. The premature demonstration and manifesting requirements mean that only older and relatively inflexible technologies for robotics and automation will be used, and any potential gains for assembly, repair, and operation will be much less than if a flight demonstration were delayed until clear-cut applications were defined and some of the newer developments could be used.

The planned early manifesting of the FTS is not currently of any real use since it cannot be relied on for assembly and will not (and should not) be on the assembly phase critical path. Its possible use for Space Station inspection purposes is of marginal benefit and does not justify its early position in the assembly phase launch sequence.

\section{Assembly Phase Utilization}

The main activity during the assembly phase will be the deployment and checkout of the Space Station and its systems. As noted earlier, the committee believes that the assembly sequence is over constrained, leading to a long-term penalty in terms of laboratory outfitting, utilization, and Station operations. For example, the life science experiments in basic gravitational biology and in preparation for extended manned missions require extensive use of several large facilities on the Space Station. These major facilities have been strongly recommended by a series of NRC and NASA advisory committees, and include specimen-holding facilities, bioisolation work stations, and a centrifuge. The current plan, which does not accommodate the animal/plant centrifuge in the U.S. laboratory module, and which proposes to assemble and check out the life sciences facilities on orbit, does not appear to meet the requirements established by the committees. The removal of the centrifuge from the U.S. laboratory to a node, which may or may not be able to properly accommodate it, is of concern: The separation of the centrifuge from the specimens, cage cleaner, work station, and general laboratory tools creates operational problems, and does not appear to be warranted by concerns over vibrational disturbances in excess of those caused by crew motions. In addition, the installation and checkout of the centrifuge on orbit is likely to prove difficult to accomplish.

Finally, the committee believes that, to the extent that the overall Space Station assembly and checkout activities allow, the early, man-tended configuration of the Space Station could be used for meaningful life science and materials science 
microgravity experiments. In particular, potential life science activities might range from short-duration verification of specimen holders to longer term biological investigations of the influence of weightlessness on cells and microorganisms. However, the committee notes that the proposed launch of the U.S. laboratory module with only materials sciences facilities should be reconsidered, as it would preclude the above-mentioned life science research activities.

\section{MATURE UTILIZATION AND OPERATIONS REQUIREMENTS}

Once the assembly phase has been completed, the Phase 1 Space Station will enter its period of mature utilization and operations activities. The committee identified a number of issues pertaining to the requirements associated with these activities.

\section{Microgravity Environment}

The Space Station design reflects the emphasis on providing a volume within the U.S. laboratory module, and later within the Japanese Experiment Module (JEM) and Columbus modules, in which experiment racks experience a very low (on the order of $10^{-6}$ g) steady-state acceleration. A particularly

sensitive issue to both the Space Station life science and materials science users is the production, transmission, and absorption of vibrations at frequencies that could damage the microgravity processes under study. Until the Space Station program tackles the vibration transmission issue squarely (including provision of structural damping within and between modules), crew-induced disturbances, which are inherent to any manned spacecraft, as well as the additional vibrations from fans, pumps, valves and centrifuges, will have to be treated empirically--with a resulting awkwardness in layout and operations. The committee believes that, as part of resolving the vibration transmission issue, the additional analysis should be done to determine whether responsibility for providing vibration isolation at the rack level of certain experiments should rest with the Space Station program or with the individual experimenter.

In general, the provision of a very low steady-state acceleration environment will require careful, continuous tracking and control of the center of gravity. However, 
apparently little attention has been given to the possibility of continuous reboost, using the resistojet thrusters, in order to overcome the effect of the $3.0 \times 10^{-7} \mathrm{~g}$ average

atmospheric drag expected at the planned Station altitude of approximately 220 nautical miles. The drag necessitates a periodic reboost to maintain the Station's orbital altitude. Periodic, as opposed to continuous reboost, could necessitate taking acceleration-sensitive experiments off-line during the reboost interval, which would have a negative impact on some user experiments. However, there are concerns relative to continuous reboost associated with potential contamination of attached payloads, as well as with possible torque disturbances that would have to addressed. Moreover, the atmospheric drag at the nominal 220 nautical mile altitude is not steady, but is strongly affected by, among other things, the diurnal bulge, seasonal variations, and solar storms. Over the span of a few orbits, the drag variations can exceed a factor of ten, with limited predictability. While the committee did not have time to examine in detail the trade-offs involved with continuous reboost, it feels that the concept has sufficient merit to warrant evaluation by the Space Station program.

\section{Crew Utilization}

On-orbit crew time will be a scarce resource, given all the conflicting demands made on it. The amount of crew time available to users is estimated to be about 30 percent of total time on orbit. However, this estimate was made prior to detailed studies of system maintenance, operation, and repair requirements on available crew time. In addition, extended duration crew operations (EDCO) activities--medical studies to verify the feasibility of long crew stay times on orbit--are planned to take up 4 percent of the available crew time. Moreover, extravehicular activity will have a significant time overhead. The committee believes it is important that the amount of crew time available for user activities be protected against erosion as maintenance and operations requirements are better defined.

One important way that the impact of scarce crew time for user activities can be minimized is through remote monitoring and control of experiments by ground investigators. Thus, the committee believes that two-way data and voice-video communication to the user laboratories, taking advantage of the new telescience developments, must be designed into the 
communication network. The use of these systems should then be incorporated into the operations plans. One impetus for making maximum use of telescience is that it will not be possible to assemble full payload teams on the ground to serve at the Payload Operations Integration Center for long durations. The Space Station program and the Office of Space Science and Applications need to find the technical (and financial) means to provide remote experiment monitoring and command of experiments.

Another means of increasing the effectiveness of crew utilization in support of user activities is to get Space Station scientists assigned to specific user experiment payloads as early as possible. For example, by 18 months prior to flight, a major payload has already gone through preliminary and critical design reviews, and probably through major phases of construction, as well. This is much too late in the payload preparation cycle to involve the primary operators (and repairers). Assignments should be "keyed" to payload development cycles to ensure that appropriate operations expertise is available during the early stages of design of the hardware and associated operation concepts. Attention and involvement early in the payload development process will reduce wasted time on orbit.

Coupled with the need for early crew involvement in experiment payload development is the need for a concerted effort to reduce the integration time for proposed Space Station experiments. If the Space Station is to be a useful 30-year research facility, it must be accessible for experiments and follow-up investigations on a timely basis. Furthermore, the flow of instruments and facilities onto and off of the Station must be carefully planned from a practical point of view. As was mentioned earlier, the committee is concerned that documentation, verification, and integration requirements for user payloads, if not specified and implemented reasonably, can have negative impacts on cost, time, and scientific productivity.

Finally, the committee supports NASA's current plan for Space Station crew selection and training. That plan provides for the assignment of Station operators, Station scientists, and payload scientists, with the first two categories being staffed by career astronauts and the last category being filled by researchers who are not career astronauts.

Based on the Space Shuttle Spacelab mission experience, it is important that there be a class of crew that is assigned to 
and primarily responsible to the user organizations, as the introduction of independently assigned payload specialists has provided advocacy for the user community in the crew and operations areas. The committee is concerned that post-Challenger crew selection procedures do not lead to a diminution of opportunities for independently assigned Space Station payload scientists. Furthermore, the committee believes there is merit in having the career Space Station scientists assigned and evaluated by appropriate user organizations, since the scientists' prime duty is the support of payload operations.

\section{Commonality}

The science users of the Space Station all desire standardization, and where possible, commonality of equipment both across disciplines and across modules. The Space Station should not be limited to a set of fixed and inflexible protocols, but should resemble a ground laboratory in which new pieces of equipment are brought in to replace or supplement other pieces as an experiment matures or as new and unexpected data is produced. Standards for power, video, data, and fluid coupling should permit and encourage the interchange of equipment and minimize the need for spares within the entire Station.

The committee believes that the Space Station program should not rely upon the possibility that certain common manufacturers might be called upon to supply all modules with equipment to reach such commonality (the approach briefed to the committee). The consequences of failing to achieve commonality are significant: Imagine the frustration of an astronaut who needs a special lens, available only on a video camera in another module, but who finds that the lens connectors are not compatible and that the camera on which the lens does fit is at another video standard than the recorder in the rack where he or she is working!

\section{Logistical Resupply}

Logistical support for a 30-year, orbiting research facility will be a complex undertaking, with a range of associated issues. Time limitations did not allow the workshop committee to examine this area in great depth, but it did identify a number of issues that it believes need to be addressed. 
The Space Station program has no provision for unmanned resupply of the U.S. modules (e.g., facilities for unmanned rendezvous and docking). The 1987 NRC Committee on the Space Station recommended that "NASA should make plans for eventual logistical support of the Space Station with expendable launch vehicles, as well as with the Shuttle" (Report of the Committee on the Space Station of the National Research Council, p. 7, 1987). The workshop committee agrees with that recommendation.

Furthermore, the flow of instruments and facilities onto and of $f$ of the Space Station must be carefully planned from a practical point of view. As was mentioned earlier, the committee is concerned that documentation, verification, and integration requirements for user payloads, if not specified and implemented reasonably, can have negative impacts on cost, time, and scientific productivity.

The committee also feels that NASA should continue to examine how much trash will be returned from the Space Station by the Shuttle versus disposal by controlled burn-up of trash canisters during reentry into the atmosphere.

Finally, the committee notes that the current design has no life-support provisions for animal and plant specimens during ascent to the holding facilities in the Space Station laboratory or during return to earth. This requirement is complicated by the scientists' requirement for late access to the animals before launch and prompt removal of them on orbit and on their return to earth. The committee is concerned that there appears to be no focal point for aggressively dealing with this item, despite its importance.

\section{Contingency Planning}

The workshop committee believes that contingency planning has progressed in a very positive sense over the past year. There still is an enormous amount of work to be done in the area, but the results to date appear to be the outcome of an established process--one that was not in place at the time of the 1987 NRC Committee on the Space Station's study.

As an example of the situations that involve contingency planning, the assembly of each of the Space Station elements will be constrained. The 100 percent contingency time for extravehicular activity during assembly flights appears to provide an adequate margin for now. However, past experience has shown that anomalies can occur that might exceed this budget, such as was demonstrated on the "Solar Max" retrieval 
mission. The availability of the extended duration orbiter configuration for each of the Shuttles should be considered to enhance the probability of completing the deployment sequence in the event of contingencies. However, the committee recognizes that use of extended duration orbiters imposes a weight penalty on the amount of payload that can be delivered to orbit by each Shuttle. The committee believes further analysis of this subject is needed.

\section{EVOLUTIONARY PHASE REQUIREMENTS}

The Space Station program properly and commendably provides accommodations for future sof tware and hardware ("hooks and scars") to permit ready modifications and growth as the Station evolves. This is an essential feature of the design that must be retained; not all future evolutionary needs and contingencies can be anticipated.

\section{Essential Evolutionary Capabilities}

It appears inevitable that successful operation of the Space Station will stimulate demands to (1) increase available electrical power, (2) add internal and external laboratory facilities, (3) increase crew size, (4) improve flexibility of operations, (5) incorporate new technologies, and (6) add volume for improved habitability. These potential demands appear to be accommodated by the current design, and must be protected during the development.

It also appears that efficient, mature operations near the manned base will require transportation capabilities not available during the assembly phase. One option is the space-based orbital maneuvering vehicle. The capability to add such capabilities should be protected.

\section{Heroic Evolution}

Visionaries will likely always imagine more revolutionary futures for the Space Station than can be attained by the evolutionary process discussed in the preceding paragraphs. Among the possible futures are the use of the manned base as a staging facility for human interplanetary flight or the deployment of a major microgravity factory in space. 
While the above-mentioned examples may be legitimate aspirations, their influence on the design of the current Station must be tempered by the uncertainties of the missions, their affordability, their likely deployment schedule, and other considerations. There is much to be learned about space research and operations in the current Space Station design and its expansion using the planned "hooks and scars." It is not evident, however, that 20 years hence the current design will be the most cost effective or technically appropriate for major new functions. It could well be that a more advanced facility would be needed. Nonetheless, evolutionary capabilities must be retained in the design of the Space Station, and NASA appears to be taking steps to see that this occurs.

The committee cautions that the incorporation of heroic evolutionary capabilities should not be permitted to add excessive complexity, increase costs, or extend the deployment schedule of the current Space Station, and supports NASA's continued vigilance in this regard. 


\section{Issues Related to Systems Requirements and Design}

The committee devoted considerable time during the workshop to the identification of issues pertaining to the major systems of the Space Station. Those issues are discussed in this chapter. As was noted earlier, this is not an exhaustive set of issues; the time available did not permit that level of review. Furthermore, the presence of expressions of approval for particular system and subsystem design decisions and their absence for others should not be taken as overall evaluations of a subsystem's acceptability in the eyes of the committee.

In the course of its review of subsystem requirements and design approaches, the committee identified a number of issues related to the interactions and interdependencies of Space Station subsystems. It believes that more attention needs to be given to such overall "architectural" considerations. The committee's concerns are summarized below, followed by a discussion of specific subsystem issues.

\section{SYSTEMS ARCHITECTURAL CONCERNS}

\section{Systems Requirements}

Requirements for systems and subsystems should be traceable to their source. In this phase of the Space Station Freedom design process, many things are changing, and there will likely be experiments or operations in the future that are not well defined at present. Therefore, some requirements will be based on well-understood needs and others will be present to insure flexibility for the future. Nonetheless, there should be a clear basis for why size, weight, power, or other design requirements have been specified and an understanding of how 
systems might be simplified, reduced in size, or changed in a favorable way if other systems or experiments change. The committee did not find as ready an awareness of these considerations as it believes is desirable at this stage in the Space Station development program.

\section{Systems Interactions}

As an example of the interactions encountered at the system level, consider the controls, structural dynamics, and propulsion systems. These systems have each been successfully planned to meet the initial design requirements. The next step should be to see in what ways the experience obtained in the design process can be used to improve the engineering of each system to simplify the designs or help in overcoming any problems that remain.

Attitude control and structural dynamics always interact. In the Space Station, there will be disturbances, the response to which will be influenced by structural dynamics. For example, astronauts will be exercising and moving around in the Space Station; the Station's response to their movements will be amplified by the structural resonances, thus causing disturbances to the microgravity experiments that may be two orders of magnitude above the desirable level of $10^{-6} \mathrm{~g}$.

The baseline design control bandwidths are quite low--about a factor of 10 lower than the lowest structural mode frequency of $0.2 \mathrm{~Hz}$. This permits relatively simple control laws. Structural damping is expected to be sufficient to give the significant modes a damping ratio of 0.5 percent. This ratio is said to be adequate to insure control behavior that meets the requirements. However, increased damping would permit further simplification and accommodate future configuration changes without requiring major control system changes. Increased damping also would reduce the response in the microgravity laboratory areas to astronauts' movements.

Thus, structural changes should be considered an available design option to further simplify control of the Station and to partially attenuate the effect of a disturbance. The resolution of the astronaut exercise problem will probably require other measures as well, such as isolation of the exercise equipment, changes in the orientation or position of the exercises, possibly a modification of the type of exercise or how it is performed, and an increase in the allowable 
microgravity levels in the laboratory, but structural damping will improve the situation and is an important part of the overall solution.

The difficulty the committee has observed is that parameters such as damping and stiffness appear to have been taken as fixed, with control techniques then developed that would be acceptable under the specified conditions. Alternatively, one could have a range of control capabilities being used to specify structural parameters. Damping, stiffness, and mode slopes at the location of the integrated sensors and control moment gyros are examples of parameters that could be specified as goals that would let the design of the structure simplify or improve the total control/structural resonance/vibration excitation picture.

It appears currently that each system and subsystem designer is simply accepting a model and proving it can meet requirements. The committee believes that better communication between designers with a common goal of improving the overall system would produce a better design. They should be required to find parameters such as damping that have beneficial effects on several other systems and to learn quantitatively what the cost would be across systems of achieving improvements. In the present technical management environment there does not seem to be any way of getting a system designer (e.g., for structures) to make a change unless a requirement is not met.

There are precedents for such an approach. One European contractor has intentionally introduced damping in the joints of a spacecraft structure to provide such conservative design margins that a costly structural analysis is not needed each time there is a configuration change. Proposals have been made to include damping in each strut used in the Space Station, but since these proposals apparently have not been funded, their cost in weight, volume, and dollars is not known. By analogy, the committee believes that other parameters that affect several systems should also be evaluated.

\section{Allocation of Systems Requirement Impacts}

The workshop committee is concerned that the impacts of specific system requirements have not been clearly allocated. For example, sizing of control and propulsion components was not always traceable. It would be helpful to know what disturbances or maneuvers have determined the control authority. In some cases a single experiment may have been the 
determinant, and in others the need to assure flexibility. In each case, an experiment or a general requirement should be "charged" in the overall design process with the escalation in size, weight or power, and so on, it requires. For example, waste disposal of a noxious gas requires storage, with the gas then used as a propellant for a resistojet. Continuous release of waste gas would be possible except for the requirements of some experiments with special viewing needs. These experiments need to be charged for the extra mass, complexity, and increased risk associated with having to store the noxious gas.

\section{Modeling}

The Space Station will be larger and of a different technology than previous spacecraft. Initial mathematical models are not likely to be accurate representations of the Space Station'sdynamic structural behavior. Each stage of construction should be used to learn how to better model the behavior. More than a dozen successive spacecraft (protostation elements)--each an extension of the previous one--will be instrumented to provide help in understanding the nonlinearities and damping (typically not well modeled) as well as mode shapes and frequencies.

Assumed Space Station damping is 0.5 percent, for example, whereas some estimates of what may be found have been as low as 0.003 percent. All modes are assumed to have the same damping, although this is never true. This characterization is important for robust control (i.e., providing a control system that will work based on a design model different from the behavior of the actual vehicle) of an evolving spacecraft during construction, when the configuration changes with the arrival of a transport vehicle (initially the Shuttle), and when an experimental payload or module is added or deleted.

Controls and structures engineers plan to utilize the data from the interim spacecraft and are developing analytical tools to assist in the data reduction. For the data to be useful, the allowable turnaround time must be very short. The committee believes that special attention should be paid in the development schedule to optimizing the use of each spacecraft so that data can be applied to the next launch package. 


\section{Growth Flexibility}

Future configurations are being considered for the Space Station. Accommodations for future growth are included in the design. However, the evaluation of system and subsystem changes required to accommodate this growth does not appear to have proceeded apace. Each future configuration need not have detailed system and subsystem designs, but the committee recommends that flexibility for future modification and growth be given adequate weight in the design process.

\section{SPECIFIC SYSTEMS ISSUES}

The following sections identify issues and concerns raised by the committee with respect to specific Space Station systems.

\section{Software and Data Management}

\section{Overview}

The Space Station sof tware and data management systems are highly complex. This complexity appears to be necessary to meet the many requirements placed on the Station. These requirements in turn dictate that the Space Station will have many systems and subsystems, with many interactions between them (ranging from preventing any interactions between specific systems to allowing high-bandwidth access). The on-board sof tware will constitute the largest on-board space software and data processing system ever developed. Likewise, the ground systems will be among the most complex ever designed and developed.

Major software risk factors include the following:

- The scope of the software is unprecedented (at least at NASA), and no prototypes exist.

- A new sof tware architecture is being proposed for flight sof tware.

- A "waterfall" methodology has been inherited (i.e., first write volumes of requirements, then implement, then integrate, test, etc.) but not evaluated.

- The requirements will not be clear until much later in the Space Station's development. 
- The flight software, for example, is quite large (about 800,000 lines of code).

- The software is being developed by multiple

(approximately ten) noncollocated contractors.

- The schedule calls for rapid development.

- No risk management plan exists.

- The software is of a high level of criticality.

In general, NASA is to be commended for recognizing the criticality of software and data management to the Space Station program, and for taking a proactive approach to addressing software-related issues. Some particularly good examples of this proactive approach include the following:

- The investment in a program-wide Sof tware Support Environment (SSE) and a Technical and Management Information System (TMIS).

- The proactive stimulation of SSE user groups, Data Management System (DMS) working groups, and early sof tware requirements analysis.

- The open system architecture for the DMS, and the early definition of key standard DMS elements (Ada, Intel 80386 instruction set architecture, ISO/OSI network protocols).

- The definition and development of the Multi-System Integration Facility and the Data Management System Kits to aid in hardware-sof tware integration and verification.

Nonetheless, the committee is concerned about a number of aspects of the Space Station software and data management capabilities. Successful sof tware development is characterized by well-understood, well-defined, and stable sof tware requirements; elimination of high-risk software items; precisely defined interfaces between the sof tware components; and a mature, in-place SSE. As mentioned above, NASA is cognizant of these conditions, and has initiated a number of commendable efforts to achieve them. However, the current process model, early schedule, and available resources early in the development activity are not well matched to the challenge. The most significant sof tware and data management issues and concerns identified by the committee during the workshop are discussed below. 


\section{Sof tware Risk Management}

The most effective technique for achieving the above conditions is development of a sof tware risk management plan, which identifies the key sof tware risk items and establishes the necessary schedules and resources for resolving the risk items by the software Preliminary Design Review. Aspects of Space Station sof tware likely to have risk associated with them include the following:

- Real-time performance, particularly in crisis mode when there are more active users, more transactions per user, and more complex transactions.

- Distributed operating system and Data Management System development, particularly in the areas of reliability and fault tolerance, efficiency, deadlock avoidance, and database integrity (e.g., design of database locking mechanisms).

- Information security and privacy assurance.

- Use of Ada features. The committee believes Ada is the right language to use for programming, with the caveat that Ada not be mandated for possibly inappropriate parts of the life cycle, such as requirements, specifications, and prototyping. There are particular potential risk items to address, including use of tasking in distributed, performance-critical, secure processing; use of exceptions and elaboration in reliability/ availability-critical situations; adequacy of compilers and run-time support capabilities for very large software programs. Selective nonuse of Ada in some special programming situations (e.g., artificial intelligence) should be considered.

- User interfaces. Besides user-friendliness and user interface consistency across applications, this also includes hardware/sof tware systems engineering to avoid equipment overkill (e.g., CRTs that soak up large quantities of scarce electrical power).

- Software development schedules.

A particularly effective risk resolution capability for sof tware design uncertainties is rapid prototyping. Early use of rapid prototyping in such areas as user interface systems, distributed processing, and critical algorithms should be much more strongly emphasized. 


\section{Sof tware Development Schedules and Uncertainties in Application Software Requirements}

The review of most of the Space Station systems (communications and tracking, fluid management, thermal control, electrical power, life support, crew operations) indicates that major uncertainties will exist for quite some time in the operational requirements that the software is intended to support. In such a situation, an early target for baselined sof tware requirements is unrealistic. A preferable approach is to have the sof tware organizations prepare draft designs and prototypes of the critical sof tware applications support capabilities, and iterate these with the applications designers in order to converge on a set of realistic and well-matched sof tware requirements somewhat later in the development cycle. This approach would also provide more time for SSE- and TMIS-support capabilities to mature, as discussed next.

In general, the current sof tware schedule emphasizes early completion of software requirements documents, which then become baselined and more difficult to modify. The early schedule preferably should emphasize execution of risk management plans and resolution of risk items before sof tware requirements are "cast in concrete."

Finally, it appears that the first item in the critical path for the sof tware is the DMS. As discussed earlier, it is necessary to begin the prototyping process for Space Station software as soon as possible, in order to allow convergence of design, to satisfy safety and supportability needs, and to keep costs in line. Two concerns arise. First, a decision on the DMS operating system might not be made until the third quarter of 1989 , which would be a serious problem since it potentially delays by one year many of the sof tware prototyping efforts. Second, criteria for making the decision were not evident to the committee from discussions with the NASA briefers.

The committee believes that the major criteria should include early and wide availability to sof tware developers (including experiment developers). This availability would allow all developers to begin as soon as possible, reduce training costs, increase the likelihood of reliability, and so forth.

An effort should be made to establish the operating system, or at least the baseline from which it will evolve, as soon as possible so that prototyping can begin. 


\section{Software Support Environment}

Given that the SSE is on the critical path of all sof tware development, it is particularly important to avoid risks of SSE functionality, schedule, performance, and robustness problems. Among the key candidate risk items that need to be defined and monitored in an SSE risk management plan are the following:

- Performance scaling of the SSE to support the development of very large sof tware complexes.

- Consistency of separately developed rules and tools (e.g., object-oriented and Yourdon-De Marco approaches).

- Provision of tools to support design, development, and testing of distributed Ada software.

- Structure and performance of the project data base and data base manager.

- Configuration management and version management models and support.

- Prioritization of incremental capabilities to project needs.

- SSE proliferation control.

- Consistent user interface across dissimilar workstations.

Even if the SSE is fully successful, that should not be taken to mean that the Space Station sof tware problems are thereby solved. Other sof tware risk items such as requirements definition and stability, design insight, and sof tware management effectiveness are orthogonal to the SSE's contributions.

The committee would also point out that allocation of adequate early resources is critical to ensuring that the SSE and the TMIS are capable of supporting the early software development phases, thus ensuring the commitment of software projects to full and effective use of SSE and TMIS. Particular needs are Ada-compatible requirements and design aids and applications-level TMIS data definitions.

\section{Sof tware Design for Supportability}

It does not appear that supportability is being adequately emphasized in the Space Station sof tware design approach (e.g., having sof tware requirements documents include a section on primary sources of requirements growth and change). Given the 
dominance of software support costs over development costs, techniques for enhanced supportability should be emphasized. An example of one of the most effective techniques is Parnas's "information hiding" technique, which involves identifying in advance the primary sources of change in the sof tware requirements, and encapsulating or hiding these sources of change (e.g., changes in display devices) within single sof tware modules (e.g., display driver module).

\section{Software Integration and Verification}

The committee believes that the emphasis on commonality for the Space Station is good, but that it will create additional complications and schedule pressures during integration and validation (for both sof tware and hardware). Specifically, any time a fault or inadequacy is identified in a common component, it will need to be traced back to all systems using the component; any changes made to the component must then be fully coordinated with all of the systems using it and the appropriate regression tests established and executed. This could become a cumbersome, time-consuming, and error-prone morass without appropriate change management channels and effective information system support. The change management information support capability needs to address issues involving support of common components.

Similarly, resolving concerns on export control of U.S. sof tware capabilities (e.g., SSE) and international noncommonalities (e.g., European Space Agency's separate Sof tware Development Environment) could consume an inordinate amount of management and calendar time. It may be better to press for a conservative but early and definitive set of international software working arrangements--perhaps use of separate SSEs--that would support a common set of requirements and design specification languages, programming standards, problem report formats, and reusable sof tware component descriptors.

Finally, current Space Station software integration plans emphasize postcoding activities. The Ada programming language enables software developers to begin integration during the design phase, by thoroughly defining Ada package specifications and verifying their consistency with other Ada package specifications via the Ada compiler. This capability can be a major source of improvement in software productivity and quality, and should be emphasized in Space Station software plans. 


\section{Space Station Information System Services}

In the committee's opinion, the coordination of the Space Station Information System (SSIS) with the other data distribution systems proposed by the NASA user organizations (i.e., by the Office of Space Operations and the Office of Space Science and Applications) does not appear to be very well defined.

The SSIS will take Space Station data and transmit it to the ground. From there it would be up to the user to distribute the data and/or archive it. The Office of Space Science and Applications has plans to provide a Space Applications Information System, and the Office of Space Operations has plans for a Customer Data Operations System. The plans for these systems are still being discussed. In order for a data distribution system to be truly user friendly, it must serve its users. For example, it must make the data available to the users at their home laboratory. Current public networks work mostly on packetized data transmission. This does not always produce the most rapid routing or time-synchronized data. It will be especially important during the conduct of an experiment to have both timely data and command relays (within a time frame of seconds) as well as time-synchronized data. Although technically not an Office of Space Station responsibility, the implications of not coordinating the different data distribution systems are great.

The committee also is concerned about how the Customer Data Operations and Space Applications Information Systems will interact, either with each other or with the SSIS. It will be very important to insure that users are supported at their home institutions. For example, video data will be downlinked in digital format and apparently restored to an analog signal on the ground. It is not clear how those data will either be sent to users or be recorrelated with other digital data. The additional complexities of having a single clearinghouse for commands, command checking, or transaction management underscore the need for continued emphasis on coordination in this area. 


\section{Communications and Tracking}

\section{End-to-End Considerations}

The following comments are directed at steps that need to be taken in the near future in the area of communications and tracking, as opposed to being a criticism of past steps that have or have not been taken. The early state of development of the Space Station makes it inevitable that gaps exist, but it would be of concern if the principal ones were not closed soon.

In communications and tracking, no end-to-end perspective was evident or presented to the committee. No analyses of end-to-end paths, link capacities, availability, blocking, queuing delays, and reliability were presented or referenced. The complexity of the Space Station program requires continuing analyses of all links from their origin to their final destination, including command and control as well as experimenter data links. For example, signals should be traced from the source through the Space Station's internal processing steps and channels, Tracking and Data Relay Satellite System (TDRSS) space segment, and the TDRSS ground segment to the final data destination (whether a NASA Center, an investigator, or some other domestic or foreign site).

Furthermore, the committee recommends that the Design Reference Missions should be used to test the complete communications and data system concepts to ensure end-to-end compatibility and performance. The full utility of the Space Station can obviously be gained only through the establishment of an environment rich in communications and computational capabilities for crew, operators, and investigators.

Another issue of concern to the committee is that all communications to and from the Space Station will flow via the TDRSS. While it is expected that the system will be fully operational in the Space Station era, there is a lingering concern that an alternative data path to and from the ground should be provided. At a minimum, this path should permit voice transmission and computer up- and down-loads under all circumstances. Many communications facilities around the world could be used in an emergency. They operate at UHF, S-, and $X$-band frequencies and can readily respond to the narrowband communications requirements mentioned above. The Space Station international partners would be obvious allies in such a venture, as would the foreign Landsat ground stations, which 
have long had ties to the United States. U.S. military stations could be used as well.

The committee believes that robust system operations require that interoperability of U.S. and foreign space and ground segments must be assured in both primary and backup modes. With respect to radio frequency compatibility, the Space Station is almost entirely dependent on the TDRSS. Many ground stations are available that could provide emergency backup communications directly to and from the Space Station. However, the planned foreign data relay satellites, which have not yet been manufactured, are already planned to be incompatible with the U.S. system. Assuring compatibility would be the most economic means to backup the TDRSS, enhance the total transmission capability, more efficiently deliver data to foreign partners, and close the zone of exclusion. A radio frequency compatibility plan should be developed with each of the foreign partners. The plan should include current elements, as well as proposed elements such as the European and Japanese data-relay satellites.

Furthermore, in the conduct of experiments on the Station, it is important to retain the option to transmit uncompressed, full-motion video signals to the ground. The currently proposed field/frame deletion compression technique is an unrecoverable approach suitable for broadcast television, but not for scientific research. Rather than requiring the users to supply their own digitization equipment to circumvent this difficulty, the Space Station equipment should provide either option.

\section{Electromagnetic Interference}

The Space Station obviously is dependent on many communication links for its successful operation; it is equally obvious that the vulnerability of these links to deliberate or inadvertent interference or access will be a continuing concern. Similarly, the various connected components of the Space Station contain many potential sources of interference that must be shielded from one another.

Many of the communication links on which the Station will depend operate at $\mathrm{Ku}$-band frequencies. These frequencies are also used by terrestrial networks, telecommunication satellites, and high-power direct broadcast satellites. Further, it is projected that hundreds of thousands of very small aperture terminals (VSATs) will be deployed for business 
communications. All of these sources raise potential interference issues. It should be noted that the Station's use of these frequencies is on a secondary, as opposed to a primary, basis.

History shows that many unexpected electromagnetic interference problems are first encountered when a new spacecraft is assembled. Eliminating these problems is of ten a tedious and difficult task, even when it is done on the ground with the benefits of considerable assistance by technical staff and sophisticated test equipment. However, the manned base cannot be tested as a complete assembly until it is in orbit. The relative inaccessibility of the Station and the relatively meager resources that will be available to the crew heighten the need for special attention to this potential problem.

One of the more troublesome forms of electromagnetic interference is the generation of passive intermodulation products. While this traditionally is an antenna design problem when the transmitter and receiver share the same aperture, it can also occur when there are nearby elements beyond the antenna that are illuminated by high-power sidelobes. The extended nature of the Space Station and the more than hemispheric coverage of its $\mathrm{Ku}$-band antennas raise the possibility of this problem. Again, because full-scale tests cannot be done prior to on-orbit assembly, this problem must be avoided through simulation, partial testing, and planning.

The committee believes that a number of things should be done to address the above-mentioned issues. One of the highest priorities is a theoretical--and, where sensible, experimental--examination of the vulnerability of all communication links to interference from space or terrestrial sources, including the possibility of intentional interference or unauthorized access. In addition, a similar examination is needed of the element-level (module, node, etc.) electromagnetic interference and of the element-to-element levels. It is important that a test and verification plan be developed to assure that the electromagnetic interference environment of the Station will remain satisfactory as it is assembled on orbit. Finally, a design and operations plan should be developed to assure that high-power transmissions (including antenna sidelobes) do not impinge on surfaces that may radiate passive intermodulation products back into the receiver. 


\section{Automation and Robotics}

The committee believes that the current Space Station approach to automation and robotics is commendable in its proactive approach to identification, exploration, and application of advanced automation technology capabilities to improve Space Station and crew effectiveness. Some good candidates for exploration and application have been identified in NASA's "Space Station Advanced Automation Study Final Report," May 1988. The committee has, however, identified some issues and concerns, which are discussed in the following paragraphs.

\section{Premature Flight Telerobotic Servicer Launch}

As was discussed earlier, the rush to launch a demonstration flight of the FTS concept and then to place it in service very early in the Space Station assembly process likely will have just the contrary effect to achieving the advancement in the state of automation and robotics desired by the users. Moreover, there is no clear vision of the spectrum of applications to be supported by the FTS, particularly given the existence of the Canadian Mobile Servicing System. Early establishment of the FTS product focus is essential to effective FTS design and development.

\section{Solution-Driven Versus Problem-Driven Approaches to Automation Initiatives}

While the improvement of Space Station and crew efficiency and productivity was given as the rationale for exploring advanced automation opportunities, a NASA briefer indicated to the workshop committee that "finding effective applications for artificial intelligence (AI) technolog $y^{\prime \prime}$ was a higher priority than "improving efficiency and productivity." However, most successful AI applications to date have used AI techniques as parts of an automation solution, rather than as ends in themselves. The Space Station program should press forward strongly with its advanced automation initiative and foster a synergy with the NASA Office of Aeronautics and Space Technology's automation technology efforts, but with a more problem-driven approach. Some important criteria for early AI applications to the Space Station are that they be low cost and low risk with high payoffs. Some attractive candidate areas in 
this regard are safety monitoring, housekeeping reduction, and testing.

\section{Advanced Automation Targets and Products}

The current focus of the advanced automation initiative is on providing tools and capabilities for improving crew efficiency and productivity in handling Space Station system operations. However, Space Station program functional breakouts of crew time indicated that three times as much crew time will be spent on user operations as on system operations. From a productivity-leverage standpoint, more emphasis should be directed toward advanced automation tools and capabilities to improve efficiency of user operations, and toward training designers of Space Station user applications and experiments in the use of such tools and capabilities to make their applications more crew efficient.

\section{Adranced Automation and Robotics Plan}

The Office of Space Station presented to the committee a modest plan for advanced automation and robotics, but the committee's major concern with that plan is that the total effort appears too small to address the magnitude of problems that the Space Station will likely encounter. In advanced sof tware technology, very little effort appears to be directed toward advanced automation assistance in the software life cycle. Other Space Station programs appear not to provide much help, since SSE primarily addresses acquisition of conventional sof tware engineering technology. Similarly, SSIS, DMS, TMIS, and the Operations Management System are directed toward the short term.

Particular issues that should be addressed include development of advanced tools for management of the software process, focussed especially on the problem of software interaction control, design capture, prototyping, risk assessment, and testing. In other areas such as prototyping, the technology-base support is developing in the Department of Defense, but adequate technology acquisition appears lacking in the Space Station automation and robotics effort.

Finally, a related organizational issue is that the focal point of responsibility for advanced sof tware technology development and acquisition in the Space Station program was not clear to the committee. 


\section{Electrical Power System}

The design approach of the electrical power system briefed to the committee calls for the conversion of the DC output from the photovoltaic panels to $20-\mathrm{kHz}$ power. The $20-\mathrm{kHz}$ electrical power would feed battery chargers for storage and go directly to loads through a variety of converters depending on the load. The electronic controls would consume about 15 percent of the electricity, with battery efficiency being about 85 percent. As designed, the system would be approximately 70 percent efficient from photovoltaic-generated DC power to load.

The Japanese and European programs have strongly suggested a 120-V DC power system. NASA's analysis of the "all DC" system, including DC isolation, shows losses equivalent to the $20-\mathrm{kHz}$ design. There are international political concerns involved in this issue that are independent of the engineering issues. A useful objective might be to center on the power efficiency issue and work with the international engineers to gain consensus on a power control strategy that reduces power loss and fills the users' needs. The objective should be to reduce power control losses to 5 percent. This 10 percent gain would increase the average effective power of the system from $75 \mathrm{~kW}$ to $85 \mathrm{~kW}$.

[A decision was made subsequent to the time of the workshop to convert to $20 \mathrm{kHz}$ and then reconvert to $120 \mathrm{~V} \mathrm{DC}$ at each of the three laboratory modules. The Space Station Program Office feels that the associated efficiency losses are acceptable in the interests of fostering science compatibility across modules. The committee has not had an opportunity to meet again to assess the merits of the decision.]

The committee understands that the electrical power and thermal control systems are being developed by at least two NASA sites. A total Space Station cross-cutting analysis of all power requirements (electrical and thermal) is needed. In addition, an emergency power analysis needs to be completed. It was not clear from the briefings presented to the committee what the minimum power requirements are for crew survivability or how resilient the power system will be under various failure modes.

Power adequacy is another of the committee's concerns. Current plans call for the Phase 1 Space Station to provide $75 \mathrm{~kW}$ of electrical power to all loads, with the objective of providing $45 \mathrm{~kW}$ to the experimenters. The present design of 
the Station requires at least $50 \mathrm{~kW}$ for systems operations. Moreover, all briefers indicated that system power requirements may increase beyond the $50-\mathrm{kW}$ level. The photovoltaic panel deployment schedule for the $75-\mathrm{kW}$ system has some major periods (6 to 9 months) in the installation phase during which the power installed is inadequate for the planned loads. If $75-\mathrm{kW}$ "budgets" and 100-kW "requirements" continue much longer, the committee is concerned that Space Station mission objectives will not be met. This issue appears to have both engineering and management components. One possible way of addressing the issue might be by means of a "power czar," who would review, approve, and allocate all electrical demands in the Space Station.

More generally, the committee believes that there is need for a management review of all utilities: electricity, heat, water, and so forth. The Space Station will be a small, remote community. One person in the program should be responsible for utility requirements, supply, specifications, and so on.

Management needs to develop a cross-cutting utilities oversight program with authority to raise real-time issues.

\section{Thermal Control System}

The thermal control system uses a series of closed-loop heat exchangers to control the environment on the Space Station for both equipment and people. One of the requirements on the thermal control system is that nearly $40 \mathrm{~kW}$ of heat from the power conditioners and batteries must be radiated into space. The committee believes that the impact of a DC electrical supply versus $20 \mathrm{kHz} \mathrm{AC}$ with its 10 - to $15-\mathrm{kW}$ power loss should be analyzed from a thermal control point of view.

The thermal control system programs for the thermal management of the photovoltaic power and space conditioning systems are presently at two locations: the NASA Lewis Research Center and the Johnson Space Center. Better coordination or even combination of the programs should be considered. In addition, the committee suggests that analyses be performed to address whether the photovoltaic radiators can be eliminated by one or more of the following measures: radiator,

1. moving $\mathrm{DC} / \mathrm{AC}$ converters and batteries to the main 
2. going to an all-DC system with load-specific power conditioning, or

3. using load partitioning (DC, $A C)$.

The committee also notes that it was indicated during the briefings that the $50-\mathrm{ft}$ photovoltaic radiator will not fit in the Shuttle payload bay for the first flight.

Environmental Control and Life Support System, Man Systems, and Extravehicular Activity System

Overview

The function of the Environmental Control and Life Support System (ECLSS), Man Systems, and Extravehicular Activity (EVA) System is to insure that crew members work and live in surroundings conducive to maintaining good health and performance. The success of this complex is judged by the health and happiness of the crew members as well as their effectiveness and efficiency in the accomplishment of mission objectives. The ECLSS, Man Systems, and EVA System are intimately interdependent and also are closely related to the Fluid Management System, which will be discussed subsequently. The committee identified a number of issues related to these systems that warrant attention.

\section{Microbial and Toxin Control in the Space Station}

The crew's health, and therefore the achievement of operational objectives, may be jeopardized if one or more crew members are overcome by infection or toxins. For instance, there are persisting problems in ground-based experimentation on microbes, whereby a species of Pseudomonas cannot be removed from water. Also, biocides used to cleanse the interior of space vehicles actually encourage the growth of bacteria. Another example is the heightened awareness of the Space Station as a "tight building" with the risk of accumulating indoor pollutants over the course of decades. Perhaps insufficient attention has been given to anticipating this problem and adjusting the engineering designs. If it is technically possible, a backup plan might be to exchange air by using existing interior air as a propellant.

NASA's current approaches regarding this issue are robust and tenacious. Nevertheless there seem to be sufficient 
warning signals to indicate that more must be done to alleviate the committee's concerns.

\section{In-Space Testing of Life Support Systems}

In-space testing of life support systems will help address the issue discussed above. At a fundamental level, however, more flight experimentation will be an important factor in assuring that reliability requirements for the ECLSS are met. Some components of the system are novel and untested in space. Because of the critical nature of the ECLSS, the committee recommends that key subsystem elements, such as $\mathrm{CO}_{2}$ reduction, $\mathrm{O}_{2}$ generation, and molecular sieves, be tested on Shuttle flights to demonstrate and refine their operation.

One current approach by NASA includes a plan for a 1991 simulated closed module. This is commendable but does not alter the need to do some sort of testing in space. NASA's approach necessarily places great emphasis on use of the early man-tended operations phase of the Space Station as an ECLSS test bed. Careful attention and periodic reassessments of risk during ECLSS development will be needed to make this approach viable.

\section{Medical Evacuation}

Situations are likely to arise in which Station personnel need to be evacuated after an initial medical stabilization by fellow crew members. NASA has considered the possibilities for evacuation from the Space Station under a variety of circumstances. Medical evacuation remains an issue. Medical evacuation from space imposes special logistical, training, and technical problems that will have to be solved since there is no direct experiential base on which to build. At a minimum, NASA should continue to study this issue and decide whether to prepare for a medical evacuation capability early in the life cycle of the Space Station.

\section{Human Factors and Habitability}

While not specifically an engineering concern, the committee believes that attention should be paid to the quality and quantity of time when a crew member is not at work. This attention is needed because crew members' use of free time under conditions of confinement, stress, and isolation may be a 
significant determinant of health maintenance in a situation in which crew must face heavy, important, and highly structured demands.

It is extremely important that habitability provisions, especially those that of fer comfort, privacy, and autonomy relative to crew members' use of space and time, should never be sacrificed or minimized. As an example, the findings that proper illumination may decrease depression in some people should be factored in as a consideration in the engineering design of the modules. Similarly, artificial means of designating perspective for up, down, right, and left must be incorporated in all parts of the Space Station, and indeed, NASA appears to be planning for such designations.

In addition, the committee believes that the crew's efficiency over time aboard the Space Station is an important issue. Based on experience from Antarctic research stations and the Soviet space stations, aggressive operational time lines and crew schedules may not be sustainable over long periods. The committee encourages NASA to continue its efforts to better understand this area.

\section{Radiation}

Surprisingly, there was little mention of radiation protection during the presentations to the workshop committee. Although it appears that radiation monitoring and assessment is well handled in terms of engineering design, the committee believes that it is important to continue to emphasize the need to better understand the radiation environment and the degree of protection required.

\section{Extravehicular Activity}

Besides the continuing work on decontamination (for gases and infectious agents) and consideration of astronauts' requests for a smaller EVA backpack, more development efforts are needed on monitoring for nitrogen bubbles in the bloodstream and on a means for handling vomiting while an astronaut is engaged in an EVA.

\section{FLUID MANAGEMENT SYSTEM}

The plans for an integrated fluid management facility need to be carefully developed. Although an integrated system might 
reduce weight cost and complexity, the critical nature of the ECLSS water and atmosphere systems requires that no contamination from the Fluid Management System (FMS) can be tolerated. The current design utilizes a single water and nitrogen supply system going to both the ECLSS and FMS systems. Although the design calls for check valves, the committee believes that a thorough analysis of this concept should be conducted prior to baselining an integrated system.

The committee agrees with the design concept that recycled water from urine be used only for hygienic activities and that it not be connected to the potable water system. It also agrees that the Orbiter-provided water (from the Orbiter's fuel cells) should go into the potable system as a first priority.

The issue of controlling waste liquids and gases is an important one in the committee's view. The FMS will have to contain a variety of gases and waste liquids from experiments as well as normal housekeeping wastes. The committee was told that neither the Columbus nor Japanese Experiment Module incorporate a system analogous to the Process Materials Management System planned for the U.S. modules. This situation needs to be addressed vigorously and resolved so that a safe internal and external (limited venting) environment is maintained throughout the Space Station.

Finally, further investigation is needed to identify the most appropriate means of measuring and controlling trace constituents and microbial contaminants in the atmosphere. 


\section{Management Issues Relevant to Design}

The committee's statement of task focussed on the identification of engineering issues related to the design and operation of the Space Station. However, during its deliberations the committee discussed a number of management concerns that the members felt could have a substantial impact on the technical program's likelihood of success.

The management challenge posed by the Space Station program is the greatest ever faced by NASA, including those of Apollo, the Shuttle, and Shuttle recovery. Unlike the Apollo and Shuttle missions, however, the challenges related to the Space Station are not largely driven by technological considerations and difficulties.

Among the challenges of the Space Station program are the following:

1. the complexity of the program itself,

2. coordination of the domestic and international interfaces,

3. preparation for a program that is in all practical respects a permanent one,

4. reconciliation of the diverse user communities, and

5. overcoming the strong internal NASA culture.

The first four challenges are difficult and serious, and NASA management appears to fully recognize that significant attention must be devoted to them on a continuing long-term basis. The challenge of the "internal NASA culture" itself, however, has many features that make the first four challenges more difficult to surmount. More important from the committee's perspective, this internal "culture" intrinsically 
has enormous potential direct and indirect impacts on design issues and their resolution. Subsequent paragraphs outline those of the committee's observations and concerns about the challenge of the internal culture that are relevant to the Space Station program, followed by the enumeration of some additional management concerns related to design activities that were raised by the committee during the workshop.

\section{IMPACT OF THE INTERNAL NASA CULTURE ON THE SPACE STATION PROGRAM}

\section{Background}

NASA has historically divided itself into three disciplines: (1) aeronautics, (2) manned spaceflight, and (3) unmanned spaceflight. Whether sanctioned or encouraged by senior management, the divisions were and are real.

The core of the Ames, Langley, and Lewis Research Centers' activities has been aeronautics, and no attempt at diversification or appending space-oriented work to these centers is going to change their basic character. Thus, Langley can undertake advanced manned spaceflight work, Ames can address life support systems, and Lewis can be assigned the Space Station's photovoltaic power system, but these are intrinsically appendages to each centers' basic interests. More important, the line management of the centers reports through the Center Directors to the Office of Aeronautics and Space Technology (OAST) at NASA Headquarters. This reality does not indicate that the centers will do their tasks poorly, only that Space Station activities are not mainline, do not report to the management of the Space Station program directly, and may not command the highest priority at those centers.

The core of the activities at the Goddard Space Flight Center and the Jet Propulsion Laboratory has been in the space and earth sciences, with the former institution focussed more on near-Earth activities and the latter more on the planets. Goddard and the Jet Propulsion Laboratory report to the Office of Space Science and Applications (OSSA), an office at the same level as OAST. Both have responsibilities in the Space Station program, but each institution has other missions as its principal orientation. As with the aforementioned research 
centers, the Space Station activities are not central, do not directly report to the management of the Space Station program, and may not command the highest priority at these two institutions.

Finally, the lead NASA centers for manned spaceflight (Johnson, Kennedy, and Marshall) have been heavily involved in the restoration of the Shuttle to flight (and its continued operation), and report to the Office of Space Flight (OSF). These centers do, however, see the Station as mainline to their principal mission, even though they do not directly report to the Office of Space Station.

Although NASA has a good deal of experience with matrix management, the Space Station program carries the degree of matrix support to a new level. As noted above, none of the centers report directly to the Office of Space Station, and each center has major, demanding, relatively near-term programs that it must support. The difficulties for Space Station management can be considerable.

There is an additional complication. The Space Station program is managed at the same level in NASA as OAST, OSF, and OSSA. It is also dependent on still another coequal NASA organization for its communications: the Office of Space Operations.

Thus, while directly commanding none of the line manpower resources of the agency, the Office of Space Station relies on one coequal office for its launches, another for its supporting technology, a third for its payloads, and a fourth for its communications. Not to be forgotten are three foreign entities who are responsible for major modules or systems as well.

\section{Impact of Management Complexities on the Space Station Technical Program}

As a result of all of the above constraints, the Space Station management structure has generated layer upon layer of institutional and interface documents of staggering complexity. Direct management paths are few and extend only from one institutional barrier to another. Diffusion of power and responsibility is extreme. The likelihood of swift, effective action is small for any substantive matter. A missing ingredient is the cohesive attribute of strong and creative technical leadership providing management and oversight that permeates to all levels--leadership, which in industry, is associated with a chief engineer and his or her staff. 
Whether the issue is communications, launches, payload, or manpower resources, the leader of the Space Station program can only negotiate with equals; failing that, the Administrator or Deputy Administrator must adjudicate and become the de facto project manager.

The committee attributes part of the shortfall of key manpower at the Level II Space Station Program Office to the management structure and foresees that increasing tensions will mark the program if clearer lines of authority do not emerge in the future.

\section{IMPACT OF PROGRAM INSTABILITIES ON THE SPACE STATION TECHNICAL PROGRAM}

Budgetary uncertainties, certain congressional directives, and operational limitations of the existing post-Challenger Shuttle have produced program instabilities that may have increased design complexity and reduced design conservatism.

The Space Station program has been subjected to considerable schedule and programmatic turbulence due to annual budgetary uncertainties. The current schedule and budget profile may not be achievable if current budgetary instabilities persist. It is difficult to conduct an orderly development program for a system as complex as the Space Station when the amount of resources that can be allocated to development by the program management is largely unpredictable from year to year.

As was mentioned earlier, some of the congressional directives to the Space Station program appear to the committee to have increased the complexity of the development process without any compelling technical justification. An example is the removal of the life sciences centrifuge from the U.S. laboratory module to a node. While the committee is somewhat skeptical of the measure's desirability in the absence of a demonstrable technical need, its main concern is that it is very destabilizing to an already constrained program to have additional requirements arbitrarily imposed. If the discipline of the design process is to be maintained, proposals for additional requirements must go through established program review mechanisms so that their impacts can be properly assessed before the additional requirements are accepted.

Finally, the 1987 NRC Committee on the Space Station noted that "from the inception of the Space Station program, the 
Shuttle has been the sole means of space transportation contemplated for Station deployment and support. This has resulted in a Space Station design constrained by the Shuttle cargo bay dimensions and the Shuttle's weight lifting capacity. These constraints have increased the difficulty of satisfying user requirements in a number of ways.... The deployment process and the operational concept also have been influenced by the lack of other means of space transportation. For example, use of the Shuttle will require substantial levels of extravehicular activity and on-orbit outfitting of the laboratory and habitation modules. The constraints imposed by the Shuttle have become more restrictive as Shuttle capabilities have been reduced, first by shortfalls from original specifications and later by modifications required af ter the Challenger accident" (Report of the Committee on the Space Station of the National Research Council, p. 19, 1987).

The workshop committee agrees with the above observations and believes that management of the Space Station program will continue to be complicated by sole reliance on the Shuttle for the deployment and support of the Space Station. If nothing else, maintenance of the planned assembly schedule for the Station will be dependent on maintaining a predictable Shuttle launch rate and manifest through the late 1990s.

\section{MANAGEMENT OF FREE-FLYING PLATFORMS}

The committee concurs with the 1987 NRC Committee on the Space Station's observation that there is no significant scientific or operational relationship between the U.S. polar platform and potential U.S. co-orbiting platform and the Space Station manned base development. The retention of the platforms within the Space Station program further complicates a very difficult management task conducted under severe manpower constraints.

Both of the platforms may be justifiable on their own merits, but to carry these programs in the Space Station Program Office just because they have some commonality with Space Station components is not an effective use of the available Space Station program management talent. Platform development should be transferred to the Office of Space Science and Applications. 


\section{ASSOCIATE CONTRACTOR RELATIONSHIPS}

The Space Station development program faces the prospect of bogging down in organizational, managerial, and technical debates unless an accepted and authoritative contractual hierarchy is established with the contractors through their respective offices. It is of concern to the committee that a disciplined and contractually binding process for integration is not evident; one needs to be established early in the program.

The initiative taken by the Office of Space Station formally to establish associate contractor relationships is commendable. However, the problem of contractor relationships would be a difficult one even if all contractors were reporting to the same contractual authority. In the Space Station program, each associate contractor reports to a different NASA contractual authority and when disagreements arise, the companies each respond to their appropriate contracting officer. Unless the Level II Program Office can somehow gain some direct contracting authority, it will always be looking to the NASA centers to resolve associate contractor differences. If it is not possible to go to a hierarchial contract arrangement, an alternative approach might be to delegate interface control authority to the Program Support Contractor and incorporate this delegation of authority into the four associate contracts. Such a step would in no way reduce the significance of the current Level II initiative to develop good working relationships between the four major associate contractors, but hopefully would further strengthen the management structure.

\section{TECHNICAL MANAGEMENT PROCESS}

The complexity of the Space Station program demands an innovative management style that encourages streamlined action on specific design/technical issues while preserving the hierarchial structure necessary for a disciplined management process.

The present organization appears to be a top heavy, highly programmed, and time consuming management structure with no provision for getting through or around it easily or in a timely way; no technical oversight that can readily cross bounds; extremely long action times in case of unexpected 
events or emergencies; and one that is not design oriented. There is a need for streamlined paths of technical authority akin to those traditionally provided chief engineers in industry. There should also be adequate provision for someone who worries only about the technical and safety issues, resolutions, and so on, and can cross bounds as if they were not there--that is, a technical conscience or technical ombudsman. Further, the committee is concerned that the program has no adequate provision for short-cutting the technical decision-making process in special cases.

\section{SYSTEM SPECIFICATION}

A Space Station system specification is needed to contractually define the system. Although requirements are being actively defined, the framework for a system specification needs to be developed as early as possible so that responsibilities and accountabilities can be established.

In the early phases of the Apollo program, considerable emphasis and effort were placed on development of a system specification and its associated specification tree. While the Space Station program has been developing requirements, the committee noted no apparent emphasis on developing a system specification to manage the program through allocation of the specification tree segments to specific NASA organizations. A system specification developed by an organization such as the Space Station Program Support Contractor would be not only a vehicle for contract compliance but also a basis to effect change discipline and change control.

The committee's concern is that until a system specification is developed, refined, and accepted as a living contractual document, there will be confusion and uncontrolled change in space station systems, resulting in cost and schedule problems.

\section{INTEGRATION AND VERIFICATION MANAGEMENT PLAN}

Lack of a strong, crisp integration and verification management plan and architecture can cause unnecessary redesign or design compromises in the Space Station program. While there is considerable work underway in this area, there does not appear to be a management plan and architecture for integration and verification in place. 
The most obvious evidence for this conclusion is the inability by NASA to define Kennedy Space Center's verification role during the launch preparation process. The planned level of activity as briefed to the workshop committee has ranged from simple installation in the orbiter bay to total reverification of factory preshipment testing.

\section{COMMUNICATIONS AND DATA SYSTEMS}

The communications and data systems for the Station pass through elements that are developed by up to four separate NASA offices. These offices are independently managed and budgeted. Each can be affected by its own management decisions, OMB's budgetary actions, or the technical, management, or budgetary decisions of Congress. Thus, the Office of Space Station directs the development of the Space Station and its ground control centers. The Office of Space Operations is responsible for the TDRSS, its ground stations, and the communication links to the control centers. The Office of Space Flight operates the Space Shuttle and its communication links to the Station during rendezvous and docking. The Office of Space Science and Applications is responsible for many of the instruments that will be placed on the Station, data processing for its instruments, and the links connecting its facilities to those of investigators.

While there is no reason to believe that any of the above offices would act irresponsibly, it nevertheless is true that all elements must work properly with one another for the maximum utility of the Space Station to be realized. At present there is little evidence that NASA is providing adequate oversight, and the potential for errors exists (notably those of omission) even in well-intentioned, skilled organizations.

The absence of an information system functional manager at NASA headquarters could allow significant budgeting and scheduling inconsistencies that could inhibit the Space Station Information System from coming on line in a timely and cohesive way.

The committee believes that NASA should create a focal point for communications and data systems with the authority and responsibility to oversee all communication links to and from the Space Station. This responsibility should extend from data source to destination without regard to NASA's organizational divisions. 


\section{Supplementary Discussion}

The bulk of the issues and concerns discussed in this report resulted from the November 1988 Workshop at the Academies' Beckman Center. As already mentioned, the issues identified are not intended to be an exhaustive list. Neither has the report attempted to highlight all of the good features of the Space Station program--the committee's energies have been directed, at the request of the Office of Space Station, toward issue identification.

The following paragraphs outline several additional potential issues identified or further elaborated since the workshop. As the committee has not had the benefit of detailed briefings on the topics, the committee's conclusions should be considered tentative.

\section{MATERIALS}

Time limitations did not allow the committee to address the area of Space Station materials in any depth. However, the committee has some concerns that it feels deserve additional consideration. One of the most important concerns is the planned use of aluminum-clad graphite-epoxy structural elements in at least three areas: (1) for truss segments, (2) for pressure vessels at $3000 \mathrm{psi}$, and (3) for structural walls of the Logistics Module. As is already understood by NASA designers, the use of this composite material has many benefits, but it also has the serious drawback of rapidly degrading in the presence of atomic oxygen after the aluminum cladding has been pinholed. Members of the committee have heard estimates from outside sources of as low as 3 years 
exposure to the space environment before truss elements might have to be replaced (for a facility with an intended life of 30 years). NASA is planning to shield critical areas to reduce the probability of pinholing, but the committee believes that the potential seriousness of the problem requires further analysis, especially in assessing its impact on the need for additional spare parts, maintenance time, and extravehicular activity. The potential impacts of outgassing from the materials also needs to be assessed.

\section{HEALTH MAINTENANCE}

As was noted earlier, the Space Station's health maintenance facility is in an early stage of development. The facility's mission and timing, as well as its relationship to facilities for health research and extended duration crew operations activities, still need to be clarified. Moreover, the committee is concerned that all of the precursor activities required to allow design of a viable health maintenance capability may not be being adequately pursued.

For example, it is highly likely that some medical emergencies on board the Space Station would require the use of invasive techniques on humans (e.g., minor surgery, stabilization with IVs). However, U.S. experience to date in such techniques has largely been limited to drawing blood samples. One of the Spacelab flights in the early 1990s is planned to include some experimentation, mainly on animal subjects, but the committee is concerned that there will not be a sufficient data base of experience available prior to the design of the health maintenance facility and its associated operational concept.

\section{TOXIC MATERIALS HANDLING}

An issue that was touched on by the workshop committee during the NASA presentations on the Fluid Management System and the life support systems was the handling of toxic materials. The materials science research activities on board the Space Station will utilize very toxic substances. Hazardous materials handling, storage, and emergency isolation procedures developed for terrestrial laboratories are likely to 
be of limited applicability in the Space Station environment. This issue could be very significant, as its resolution is likely to have an impact on several Station system designs. The committee is aware that NASA is devoting some attention to the issue, but the committee did not have time during the workshop to assess NASA's activities in this area.

\section{POTENTIAL INCOMPATIBILITY OF USER REQUIREMENTS}

The committee is concerned about the potential incompatibilities of the requirements of different groups of users, notably between those in the materials sciences and life sciences disciplines. While NASA clearly is devoting attention to this area, the committee believes this will remain a significant issue.

\section{CREW SAFETY}

Various aspects of crew safety were discussed during the workshop and have been noted in this report. However, time limitations precluded a systematic examination of safety-related issues. Several matters of potential concern, e.g., toxic materials handling, have been identified in this "Supplementary Discussion" section. Other possible concerns include orbital debris, joint Shuttle-Space Station activities, and the overall state of emergency planning and its impact on the design process. While the committee has no reason to believe that NASA is not attaching a high degree of importance to the resolution of crew safety issues, it believes that crew safety is an area that will require continued diligence throughout the design, development, and operational phases of the Space Station program. 



\section{APPENDIX A \\ Letter from the \\ NASA Office of Space Station}

PRECEDING PAGE BLANK NUT FILMED

65

PAS 64 INTENTIONALYY BLANK 


\section{N/SA}

National Aeronautics and

Space Administration

Washington, D.C

20546

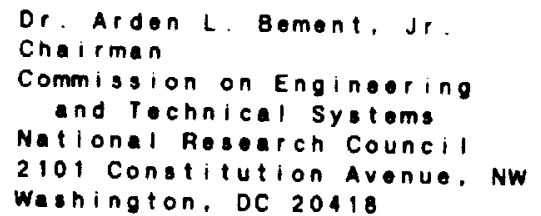




\section{APPENDIX B \\ Statement of Task}

Obiective: To explore engineering issues in the design and operation of the proposed Space Station and to assist NASA in identifying subjects that warrant special attention.

Study Plan: The Aeronautics and Space Engineering Board has been asked by NASA/Office of Space Station to form a committee of experts to conduct a planning workshop on Space Station engineering design issues. The committee will

- review the current status of the design and planned operation of the Space Station, with presentations given by NASA and other individuals/organizations as appropriate;

- identify engineering and operational issues that warrant special attention;

- prioritize the issues for NASA consideration.

The results of the workshop will be reported to the NASA Office of Space Station. 


\section{APPENDIX C \\ Presenters at the Workshop}

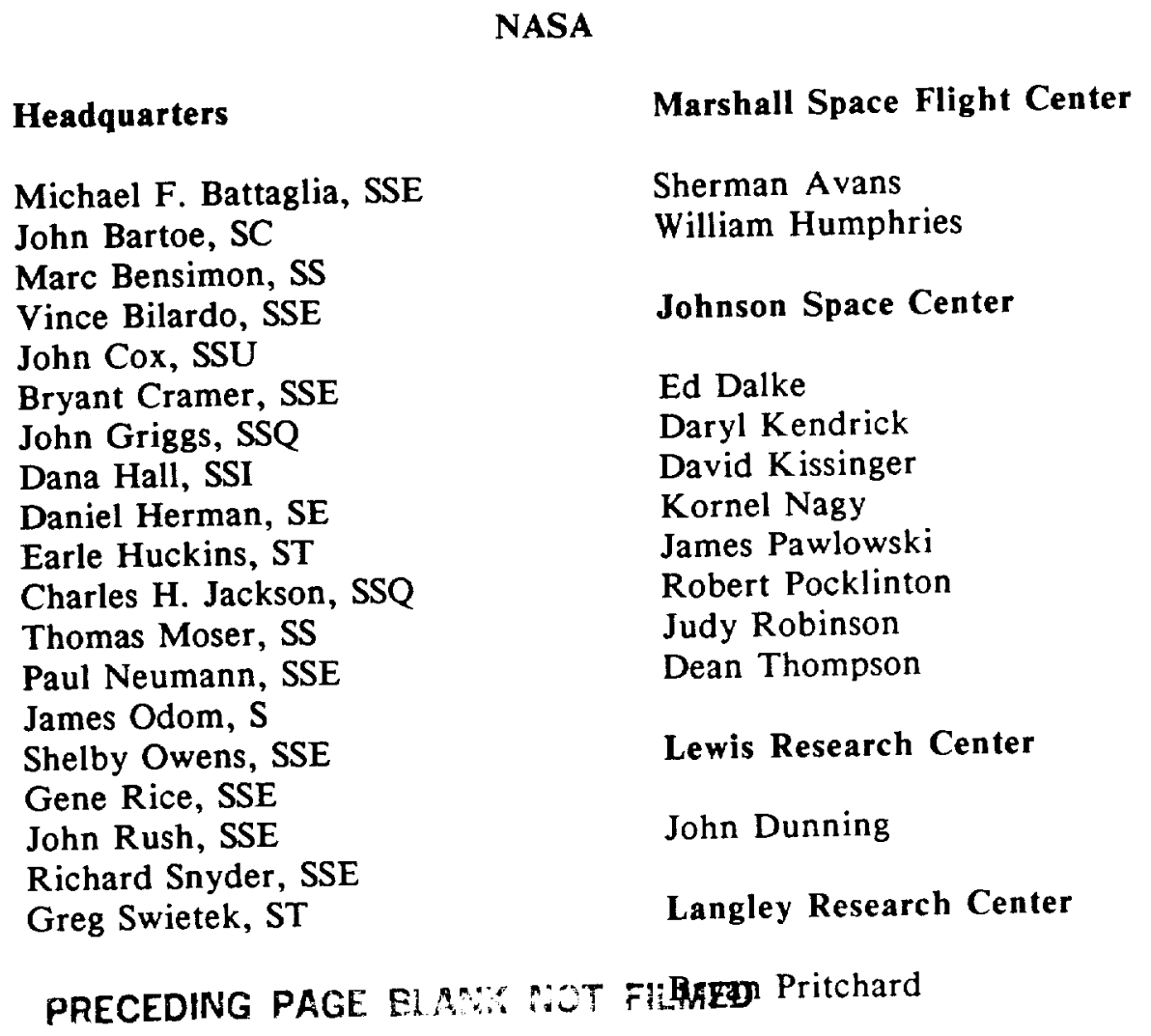

Other

George Schmidt, Booz Allen \& Hamilton

69

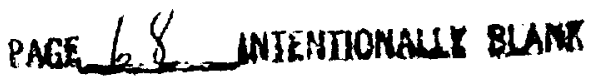





\section{Abbreviations/Symbols}

\begin{tabular}{ll} 
AC & alternating current \\
DC & direct current \\
f & frequency \\
$\mathbf{f t}$ & foot \\
$\mathbf{g}$ & gravitational acceleration \\
$\mathbf{H z}$ & Hertz \\
$\mathbf{I}$ sp & specific impulse \\
$\mathbf{k g}$ & kilogram \\
$\mathbf{k H z}$ & kilo Hertz \\
$\mathrm{kW}$ & kilowatt \\
$\mathrm{lb}$ & pound \\
$\mathrm{m}$ & meter \\
$\mathrm{Mbps}$ & megabits per second \\
$\mathrm{sec}$ & second \\
$\mathrm{V}$ & volt \\
\hline
\end{tabular}

PRECEDING PAGE BLAHK MO Finitis

71

ER $10 \quad$ INTENTIONALY BLAM 


$\begin{array}{ll}+/- & \text { plus or minus } \\ < & \text { less than } \\ > & \text { greater than } \\ - & \text { degree } \\ 10^{-2} & 1 / 100\end{array}$




\section{Acronyms}

$\begin{array}{ll}\text { AI } & \text { Artificial Intelligence } \\ \text { CERV } & \text { Crew Emergency Rescue Vehicle } \\ \text { CRT } & \text { Cathode Ray Tube } \\ \text { DMS } & \text { Data Management System } \\ \text { ECLSS } & \text { Environmental Control and Life Support System } \\ \text { EDCO } & \text { Extended Duration Crew Operations } \\ \text { EMI } & \text { Electromagnetic Interference } \\ \text { EVA } & \text { Extravehicular Activity } \\ \text { FMS } & \text { Fluid Management System } \\ \text { FTS } & \text { Flight Telerobotic Servicer } \\ \text { I\&V } & \text { Integration and Verification } \\ \text { ISO } & \text { International Standards Organization } \\ \text { JEM } & \text { Japanese Experiment Module } \\ \text { LVLH } & \text { Local Vertical Local Horizontal } \\ \text { NASA } & \text { National Aeronautics and Space Administration } \\ \text { NRC } & \text { National Research Council } \\ \text { OAST } & \text { Office of Aeronautics and Space Technology } \\ \text { OMB } & \text { Office of Management and Budget } \\ \end{array}$




$\begin{array}{ll}\text { OSF } & \text { Office of Space Flight } \\ \text { OSI } & \text { Open Systems Interconnection } \\ \text { OSSA } & \text { Office of Space Science and Applications } \\ \text { PMC } & \text { Permanently Manned Capability } \\ \text { SSE } & \text { Software Support Environment } \\ \text { SSIS } & \text { Space Station Information System } \\ \text { TDRSS } & \text { Tracking and Data Relay Satellite System } \\ \text { TMIS } & \text { Technical and Management Information System } \\ \text { UHF } & \text { Ultra-High Frequency } \\ \text { VSAT } & \text { Very Small Aperature Terminal }\end{array}$




\section{Selected Material Used \\ at the Workshop}

\section{VIEWGRAPHS}

1988. "Evolution Requirements for the SSP Preliminary Requirements Review." Viewgraphs.

Mark Craig, NASA/JSC. July 14, 1987. "Space Station Configuration Chronology (1983-1987)." Viewgraphs presented to the National Research Council.

Dr. Earle Huckins. May 19, 1988. "Space Station

Evolution--Plans and Programs." Viewgraphs presented to the Space Station Advisory Committee (SSAC).

R. Snyder. September 22, 1988. "Space Station Assembly Sequence Review." Viewgraphs presented to the Associate Administrator for Space Station Freedom.

NASA Office of Space Station. October 4, 1988. "Space Station Freedom Transportation Systems Planning--Summary." Viewgraphs presented to the Office of Technology Assessment.

NASA Space Station TMIS Task Force. March 8, 1988. "Space Station Program Technical and Management Information System Review." Viewgraphs presented to the Administrator.

NASA Office of Space Flight and the Office of Space Station. 1987-1988. "A Compilation of Recent NASA Studies on the Space Transportation Requirements for the Space Station."

Viewgraphs.

Chuck Holliman, NASA. May 19, 1988. "Orbital Maneuvering Vehicle Space Station Support." Viewgraphs. 
May 19, 1988. "Assured Crew Return Capability (ACRC)." Viewgraphs presented to SSAC.

"Joint OSF/OSS Study: ELVs in the Space Station Logistics Operations." Viewgraphs.

Senior Engineer, Office of Space Station. September 20, 1988. "Space Station Advisory Committee." Viewgraphs.

Margaret G. Finarelli. September 20, 1988. "Space Station Freedom--The International Partnership." Viewgraphs presented to the Space Station Advisory Committee.

Space Station Program. December 18-19, 1987. "Report on the Review of Space Station Systems--Reduced Cost Option."

Viewgraphs presented to the Deputy Administrator.

L. Young and C. Rudiger. March 8, 1988. "Life Science

Utilization of the Space Station." Viewgraphs.

OTHER NATIONAL AERONAUTICS AND SPACE ADMINISTRATION MATERIAL

NASA Ames Research Center. 1985. Living Aloft--Human Requirements for Extended Spaceflight.

NASA. June 1986. The Space Station--A Description of the Configuration Established at the Systems Requirements Review (SRR).

NASA. August 1987. Space Station--Science Operations Management Concepts Study.

NASA. October 1987. Space Station Operations Task Force Report.

NASA. November 16, 1987. Engineering Data Book, Vol. 1-Executive Summary.

NASA. November 16, 1987. Engineering Data Book, Vol. 2-Assembly Sequence and Configuration Definition. 
NASA. November 16, 1987. Engineering Data Book, Vol. 3-Analytical Studies.

NASA. November 16, 1987. Engineering Data Book, Vol, 3-Appendices.

NASA. March 1988. Advancing Automation and Robotics Technology for the Space Station and for the U.S. Economy. Progress Report 6--October 1987 through March 1988.

NASA. March 1988. Science and Applications on the Space Station.

NASA. April 1988. Space Station Capital Development Plan--Fiscal Year 1989.

NASA. April 1988. Space Station "Quick is Beautiful" Research Study Group--Final Report.

NASA. May 1988. Space Station Advanced Automation Study--Final Report.

NASA. October 1988. Space Station Freedom Technical Qverview.

NASA. September 23, 1988. "Assembly Sequence CR BB000468 Supporting Data."

Charts: "User Integration Scenario." Part 1: Manned Base Users. Part 2: Development of a New Platform. Part 3: Installation of New Payloads on an Existing Platform.

Charts from the "Post-Level II PRR Studies/Actions Updates as of $9 / 23 / 88 . "$

\section{NATIONAL RESEARCH COUNCIL MATERIAL}

NRC. 1985. Space Station Engineering and Technology Development--Report of the Committee,

NRC. 1985. Space Station Engineering and Technology Development--Proceedings of the Panel on Program Performance and Onboard Mission Control. 
NRC. 1985. Space Station Engineering and Technology Development--Proceedings of the Panel on Maintainability.

NRC. 1985. Space Station Engineering and Technology

Development--Proceedings of the Panel on Solar Thermodynamics

Research and Technology Development.

NRC. 1985. Space Station Engineering and Technology Development--Proceedings of the Panel on In-Space Engineering Research and Technology Development.

NRC Space Station Committee. 1986. Letter Reports from Panel Chairmen.

NRC. October 1986. Post-Challenger Assessment of Space Shuttle Flight Rates and Utilization.

NRC. 1987. Human Factors in Automated and Robotic Space Systems--Proceedings of a Symposium.

NRC. June 1987. From Critical Issues in NASA Information Systems. "User Involvement" and "Information Systems Technology."

NRC. June 1987. Guidelines for Noise and Vibration Levels for the Space Station.

NRC. September 1987. Report of the Committee on the Space Station.

NRC, 1987. Space Technology to Meet Future Needs.

NRC. January 1988. Post-Challenger Evaluation of Space Shuttle Risk Assessment and Management.

\section{PAPERS FROM 1988 INTERNATIONAL ASTRONAUTICAL FEDERATION CONGRESS}

D. Bassett, et al. "Mobile Servicing System Flight Operations and Support."

G. Bolton, et al. "COLUMBUS--Design Approach to Meet User Needs." 
R. Carlisle. "Space Station Design Integration."

R. Corban. "Expendable Launch Vehicle Transportation for the Space Station."

F. Engstrom. "The COLUMBUS Programme: Status and Perspective."

A. Esterle and A. Guell. "Analysis of Human Activities during Space Missions: Outlines of Possible Human Missions aboard COLUMBUS."

W. Evans and K. Doetsch. "Canada's Mobile Servicing System for Space Station."

H. Friedrich. "Man System Aspects in the Design Concept of the COLUMBUS Man-Tended Free Flyer (MTFF).

O. Gazenko, et al. "Main Results of Medical Investigations during Long-Duration Space Flights onboard Salyut-7--Soyuz-T."

R. Hager and H. Willenberg. "Early Space Station Laboratory User Activities."

K. Higuchi, et al. "User Accommodation Concept for Japanese Experiment Module on the Space Station."

W. Hook, et al. "Earth Transportation Node Requirements and Design."

B. Krishan. "Risk Management for the Space Station Program."

P. Kurzhals and S. Paddock. "Achieving Operational Efficiency with the International Space Station."

C. Leach, et al. "Medical Considerations for Extending Human Presence in Space."

R. Mayo. "International Interface Design for Space Station Freedom: Challenges and Solutions."

D. McKnight, et al. "The Orbital Debris Issue: A Status Report." 
G. Melkonian, et al. "Radiation Protection of Astronauts in LEO."

Y. Morishita, et al. "Program Status and Plan of JEM."

T. Moser. "Space Station Freedom--Technical and Management Challenges."

C. Pittman. "Space Station Information Systems."

G. Schmidt. "Selection of Combined Water Electrolysis and Resistojet Propulsion for Space Station Freedom."

W. Schneider. "Life Sciences Role in Systems Engineering of Space Programs."

Y. Shibato, et al. "Logistics Support of Japanese Experiment Module by the H-II Rocket."

K. Shiraki and T. Yomaguchi. "JEM ECLSS and Man System."

M. Torr and D. Torr. "The Induced Environment around Space Station."

D. Wensley. "U.S. Space Station Freedom: Orbital Assembly and Early Mission Opportunities."

\section{OTHER}

March 24, 1988. American Institute of Aeronautics and Astronautics Assessment of Space Station User Operations Plan.

American Institute of Aeronautics and Astronautics Workshop. November 1987. From The Civil Space Program: An Investment in America. "Space Station and Platforms."

Nicholas L. Johnson, Advisory Scientist. Teledyne Brown Engineering, Colorado Springs, Colorado. From The Soviet Year in Space--1987: "Man-in-Space Programs."

R. Price. October 1988. From Aerospace Engineering. "Phase 1 Space Station Power System Development." 
James L. Smothermon. May 19, 1988. "National Space

Transportation System Support to the Space Station Program."

Lockheed Missiles \& Space Company, Inc. July 7, 1988. System

Concept Document--Space Station Software Support Environment.

Rev. 2.1.

U.S. General Accounting Office. March 1988. Space

Qperations--Testing of NASA's Technical and Management

Information System. 
$-$ 


\section{NNAS \\ RNAE National Academy Press}

The National Academy Press was created by the National Academy of Sciences to publish the reports issued by the Academy and by the National Academy of Engineering, the Institute of Medicine, and the

National Research Council. all operating under the charter granted to the National Arademy of Sciences by the Congress of the Linited States. 